\title{
Immersed-Boundary-Type Models of Intravascular Platelet Aggregation *
}

\author{
Aaron L. Fogelson* \\ Departments of Mathematics and Bioengineering, University of Utah
}

Robert D. Guy

Department of Mathematics, University of California, Davis

\begin{abstract}
The formation of platelet aggregates during blood clotting is modeled on two scales using ideas motivated by Peskin's Immersed Boundary Method. The microscopic scale models track individual platelets, their mechanical interactions with one another and the surrounding fluid, their detection of and response to chemical activators, and the formation of cohesive and adhesive 'links' between platelets and between platelets and the vascular wall. These models allow inclusion of detailed mechanisms of binding-unbinding, platelet stimulus-response, and chemistry on the platelets' surfaces. The macroscopic scale models treat the same interactions in terms of concentrations of platelets and distributions of cohesive and adhesive links, and can be used to study platelet aggregation in vessels of clinical interest including the coronary and cerebral arteries. In both types of model, the development of platelet aggregates affects the fluid motion only through an evolving fluid force density, and consequently, Cartesian grid methods are effective in solving the model equations.
\end{abstract}

Key words: thrombosis, multiscale modeling, biofluid dynamics, blood clotting, Cartesian grid

1991 MSC: 65M06, 76Z05, 92C10, 92C45

\footnotetext{
ऋ Supported, in part, by grants from the National Science Foundation: DMS9805518, DMS-0139926, and DMS-0540779.

* Corresponding Author

Email addresses: fogelson@math.utah.edu (Aaron L. Fogelson), guy@math.ucdavis.edu (Robert D. Guy).
}

Preprint submitted to Computer Methods in Applied Mechanics and Engineering6 May 2007 


\section{Introduction}

Intravascular blood clots (thrombi) are initiated by damage to the endothelial cell lining of a blood vessel and involve the formation on the damaged surface of clumps of cells intermixed with a fibrous protein gel. This happens in the face of continued blood flow past the injury, and the interplay between the development of the clot and the local fluid dynamics is one of our principal concerns. Under some conditions, the clot grows to completely occlude the vessel. In other situations, it grows to a maximum size and then portions of it break away and the clot's size may settle into a rough steady state. One of our major goals is to be able to capture both kinds of behavior in our models, and to understand why they occur.

Clot formation involves two intertwined processes both of which are initiated by damage to the vessel lining. One process is platelet aggregation and begins when circulating blood platelets adhere to the damaged wall. Other platelets can be activated by chemicals released by these first platelets and then bind to the already wall-adherent platelets; this results in the buildup of a platelet aggregate or thrombus. The other process is coagulation which we view as itself comprised of two distinct subprocesses. The first of these involves a network of tightly-regulated enzymatic reactions that begins with reactions on the damaged vessel wall and continues with important reactions on the surfaces of activated platelets. The end product of this reaction network is the enzyme thrombin which i) activates additional platelets and ii) creates monomeric fibrin which polymerizes into the fibrous protein gel component of the clot. This polymerization process is the second subprocess of coagulation. Both platelet aggregation and the two parts of coagulation occur in the presence of moving blood, and are strongly affected by the fluid dynamics in ways that are as yet poorly understood. One indication of the effect of different flow regimes is that clots that form in the veins, where blood flow is relatively slow, are comprised mainly of fibrin gel (and trapped red blood cells), while clots that form under the rapid flow conditions in arteries are made up largely of platelets. Understanding why there is this fundamental difference between venous and arterial clotting should give important insights into the dynamics of the clotting process.

In this paper, we review our development of models of platelet aggregation and we point how these models are designed with the future inclusion of coagulation in mind. Thus, for example, we make provision for the later inclusion of coagulation chemistry on surfaces of model activated platelets. We describe two classes of platelet aggregation model. One involves the behavior of a collection of individual platelets interacting with the suspending fluid, the vessel

wall, and platelet activating chemicals. We refer to these as our microscale platelet models, and note that they are appropriate for small diameter arteri- 
oles and venules (approximately 50 microns in diameter), as well as, perhaps, for detailed studies of the aggregation process in small portions of a larger clot. The other, our macroscale platelet models, tracks the dynamics of the same sorts of interactions but on a larger scale appropriate for larger vessels. These continuum models involve the spatial-temporal evolution of a platelet thrombus using density functions to describe the distribution of the relevant platelets and other species. For the microscale modeling a major tool is the Immersed Boundary (IB) method. For the macroscale models, the classical Immersed Boundary method motivates our modeling approach.

Because the approach of the IB method underlies our modeling of platelet aggregation in both scales of models, we briefly review the fundamentals of the IB method. Then we describe aspects of platelet biology important in our modeling efforts. After that we describe the microscale aggregation models based on the Immersed Boundary method. Finally, we discuss how these ideas are extended to much larger spatial scales in our macroscale models.

\section{Immersed Boundary Method}

The fundamental problem for which the IB method has been developed concerns the interactions of a viscous incompressible fluid with one or more moving and/or deformable elastic objects in contact with that fluid. The motion of the fluid influences the motion of the elastic objects and vice versa, and so the IB method involves coupled equations of motion for both types of material (fluid and elastic) and solves for both motions simultaneously. To introduce the IB method we focus on a simple model problem in which a single fluidfilled closed elastic membrane is immersed in a viscous fluid (see Fig. 1). For simplicity we describe a two-dimensional model problem, but emphasize that the IB method has been used extensively for three-dimensional studies in a number of application areas. We also assume that the fluid inside and outside the membrane has the same density and viscosity (although this is not essential). The physics of the model problem is that the elastic membrane is under tension and exerts force on the adjacent fluid. These forces may cause the fluid to move and, in that case, points on the membrane move along with the fluid. In the IB method, the fluid is described in Eulerian terms through a velocity field $\mathbf{u}(\mathbf{x}, t)$ and pressure field $p(\mathbf{x}, t)$ defined at every point $\mathbf{x}$ in the physical domain $\Omega$. The elastic membrane is described in Lagrangian terms. Let the elastic membrane be parameterized by $q$, and denote by $\mathbf{X}(q, t)$ the spatial coordinates at time $t$ of the membrane point labeled by $q$. The IB equations are coupled equations of motion for the fluid variables $\mathbf{u}(\mathbf{x}, t)$ and $p(\mathbf{x}, t)$ and the membrane configuration $\mathbf{X}(q, t)$. The basic IB equations are:

$$
\rho\left(\mathbf{u}_{t}+\mathbf{u} \cdot \nabla \mathbf{u}\right)=-\nabla p+\mu \Delta \mathbf{u}+\mathbf{f}, \quad \nabla \cdot \mathbf{u}=0,
$$




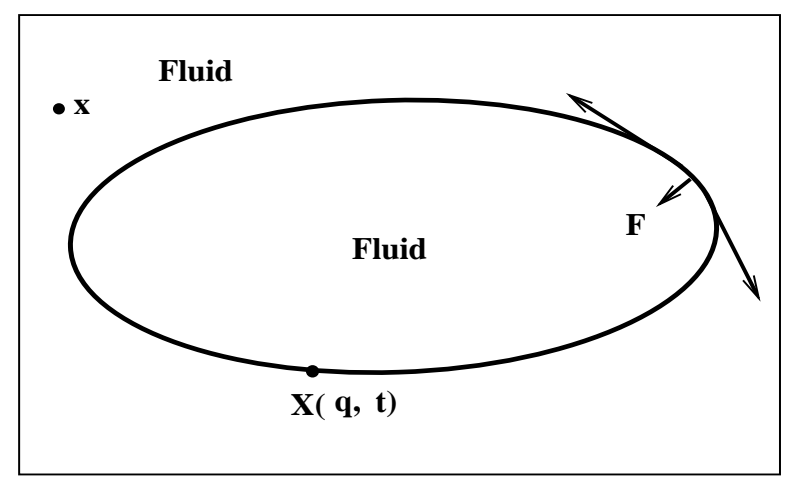

Fig. 1. Model Problem: Massless elastic membrane immersed in fluid. Here, $\mathbf{x}$ is a point in the fluid, $\mathbf{X}(q, t)$ is the location of a material point on the immersed elastic membrane, and $\mathbf{F}$ is the force generated at a point in the membrane because the membrane is stretched.

$$
\begin{gathered}
\mathbf{F}(q, t)=\mathbf{F}\left(\mathbf{X}(q, t), \mathbf{X}_{q}(q, t)\right) \\
\mathbf{f}(\mathbf{x}, t)=\int \mathbf{F}(q, t) \delta(\mathbf{x}-\mathbf{X}(q, t)) d q \\
\frac{\partial \mathbf{X}}{\partial t}(q, t)=\int_{\Omega} \mathbf{u}(\mathbf{x}, t) \delta(\mathbf{x}-\mathbf{X}(q, t)) d \mathbf{x} .
\end{gathered}
$$

Eqs.(1) are the Navier Stokes equations which describe the dynamics of a viscous incompressible fluid, of constant density $\rho$ and constant viscosity $\mu$, driven by a force density $\mathbf{f}$ which here arises because of the elastic deformation of the immersed membrane. Eq.(2) specifies the elastic force (per unit q) at each point of the immersed boundary object. The functional dependence of this force on the state of the boundary is specified appropriately to the material being modeled. An example is given below. Eq.(3) defines the fluid force density $\mathbf{f}(\mathbf{x}, t)$ in terms of the immersed boundary elastic force density $\mathbf{F}$. By integrating both sides of this equation over an arbitrary region of the fluid, we see that the total fluid force on this region equals the total elastic force along the portions of the immersed boundary, if any, that pass through this region. So the fluid force density is concentrated along the immersed boundary curve. Eq.(4) specifies that the velocity of each immersed boundary point equals the fluid velocity at the same location. This is a formulation of the no-slip boundary condition for viscous flows. The key idea in this formulation that makes the IB approach so useful in modeling biofluid problems is that as far as the fluid is concerned, the immersed objects are seen only through the force field f. Even if the objects move or deform substantially, there is no change in the geometry of the fluid region; fluid is everywhere and only the distribution of forces exerted on the fluid by the elastic objects changes. In the model problem and the platelet applications, we assume that the IB objects are neutrally buoyant; the IB membrane itself carries no mass, its mass is attributed to the fluid in which it sits.

To illustrate the specification of the IB force function, we consider the case of 
the forces generated within a stretched fiber. Let the points on the fiber be denoted by $\mathbf{X}(q)$, assume that the only force the fiber can sustain is tension

along the fiber direction $\boldsymbol{\tau}(q)$, and let $T\left(\left\|\frac{\partial \mathbf{X}}{\partial q}\right\|\right)$ give the dependence of the tension on the local stretch of the fiber. Consider a segment of the fiber corresponding to $q_{1} \leq q \leq q_{2}$ and let $\mathcal{F}$ denote the force that this segment of fiber exerts on the surrounding fluid. Because the fiber segment is massless, we have the balance of forces,

$$
0=-\mathcal{F}+\left.(T \boldsymbol{\tau})\right|_{q=q_{2}}-\left.(T \boldsymbol{\tau})\right|_{q=q_{1}}=-\mathcal{F}+\int_{q_{1}}^{q_{2}} \frac{\partial}{\partial q}(T \boldsymbol{\tau}) d q
$$

Hence, $\mathbf{F}=\frac{\partial}{\partial q}(T \boldsymbol{\tau})$ gives the force density (per unit $q$ ) in the fiber, and the force exerted on the fluid by this fiber segment is given by the integral of $\mathbf{F}$ over the segment. Specifying the function $T$, e.g., $T=S\left(\left\|\frac{\partial \mathbf{X}}{\partial q}\right\|-r\right)$ for positive constants $S$ and $r$, determines the fiber's elastic properties.

In actual IB calculations, the Navier-Stokes equations are approximated at points of a Cartesian grid placed over the domain $\Omega$. A second grid of Lagrangian points is used to discretize each elastic object, and the $\delta$-functions in Eqs.(2),(4) are approximated by smooth but very localized functions. This implies that the fluid force density is nonzero only for fluid grid points close to the immersed boundary and that the velocity of each immersed boundary point is computed as a weighted average of the fluid velocity at grid points near that IB point. Aspects of the IB method are described further below. More information about the IB method can be found in Peskin's review article [1] and the references cited therein.

\section{Biological Background}

Platelets are anuclear blood cells that have a discoid shape when circulating with the blood in their usual unactivated state. They have a diameter of about $2 \mu \mathrm{m}$ and a number density of about $250,000-300,000 / \mu$ l. They are much smaller and less numerous than the red blood cells that make up about $45 \%$ of the blood's volume, and so individual platelets have a negligible effect on the flow of the blood [2]. In their unactivated state, platelets do not adhere to one another or to the intact endothelial cell lining of blood vessels. Disruption of the endothelial layer exposes to the blood collagen and adsorbed von Willebrand factor (vWF) molecules in the subendothelial matrix. Platelets adhere to both molecules via specific platelet surface receptors. In addition to slowing or stopping platelet motion over the subendothelium, this binding triggers intracellular signaling pathways that lead to platelet activation $[3,4]$.

Platelet activation is multifaceted: (i) The platelet's cytoskeleton is reorga- 
nized and the platelet balls up into a spherical shape and extends a number of appendages called pseudopodia. The platelet becomes sufficiently flexible that over time it can spread out over the surface to which it is adhered. (ii) The platelet surface membrane changes in important ways: Integrin $\left(\alpha_{I I b} \beta_{3}\right)$ receptors embedded in the membrane are activated and become capable of binding dimeric fibrinogen molecules and multimeric vWF molecules from the blood plasma. By binding to receptors on two platelets, these molecules serve as links between the platelets. The platelet membrane also undergoes processes that makes it able to support important reactions of the coagulation process. (iii) The activated platelet secretes chemicals into the surrounding blood plasma. Probably the most important of these are ADP released from cytoplasmic storage granules and the coagulation enzyme thrombin, activated by the prothrombinase enzyme complex that can form on the surface of activated platelets. A platelet has specific surface receptors for ADP and thrombin and binding of these molecules to an unactivated platelet can trigger the activation process in that platelet. These chemicals provide a second pathway to platelet activation that does not require direct contact of the platelet with the injured vessel wall, and allows the activation process to be propagated away from the wall [5].

Exposure of the subendothelium also brings the passing blood into contact with Tissue Factor molecules embedded in the matrix and initiates the coagulation process [6]. The first coagulation enzymes are produced on the subendothelial matrix and released into the plasma. If they make their way through the fluid to the surface of an activated platelet, they can participate in the formation of enzyme complexes on the platelet surface that continue and accelerate the pathway to thrombin production. Thrombin released from the platelet surface feeds back on the enzyme network to accelerate its own production, activates additional platelets (as mentioned above), and converts soluble fibrinogen molecules in the plasma into insoluble fibrin monomers. Once formed, the fibrin monomers spontaneously bind together into thin strands, these strands join side to side into thicker fibers, and a branching network of fibrin fibers grows between and around the platelets in a wall-bound platelet aggregate [7]. Ends of the fibrin strands may be anchored to the surfaces of the platelets by binding of fibrin to integrin $\alpha_{I I b} \beta_{3}$ receptors. The coagulation process, in particular, the interactions of coagulation biochemistry with flow and platelet events, is a fascinating subject, but it is not the focus of this paper. If interested, see [8-10].

The red blood cells, which make up almost half of the blood's volume, have significant effects on the motion and distribution of platelets in blood flowing in a tube. For one, platelets exhibit much larger transverse excursions (across the tube) than under similar flow conditions in plasma alone [2]. This motion is often described as an enhanced diffusivity (above Brownian motion), and experimental studies suggest that the degree of enhancement depends on the 
flow shear rate [11]. A second effect is that the concentration of platelets is higher (by up to eight fold) a few microns from the tube wall than at other distances from the wall. This effect is seen only for shear rates above 200 $\mathrm{sec}^{-1}$ at which red blood cells deform, and, in experiments, is a function of the volume fraction occupied by red blood cells [12]. The rheological processes that lead to these observed effects are not understood; a rough picture is that tumbling and colliding of red blood cells generates local flow disturbances that lead to these behaviors. Whatever their cause, the enhanced random motion and the enhanced near-wall concentrations are likely important in determining the rate at which platelets contact the vessel wall and the rate at which wallbound aggregates grow.

\section{Microscale Platelet Aggregation Models}

Our microscale platelet aggregation models [13-16] track the motion and behavior of a collection of individual platelets as they interact with the suspending fluid, one another, and the vessel walls. These models also track fluid concentrations of platelet activating species such as ADP, cell-cell and cell-surface forces, fluid motion, and the local fluid forces on the growing thrombus. In the models, nonactivated platelets are activated by contact with reactive sites on the injured wall, or through exposure to a sufficiently high concentration of activator in the fluid. Activation enables a platelet to cohere with other activated platelets, and to secrete additional activator. The platelets and the secreted chemical move by advection with the fluid and diffusion relative to it. Each platelet and each vessel wall is represented as an IB object, i.e., as a collection of elastically linked Lagrangian points that each move at the local fluid velocity. New elastic links are created dynamically to model the adhesion of a platelet to the injured wall or the cohesion of activated platelets to one another. The multiple links, which in the models can form between a pair of activated platelets or between a platelet and the injured wall, collectively represent the ensemble of molecular bridges binding real platelets to one another or to the damaged vessel. The links exert forces on the surrounding fluid to resist motions which would otherwise separate the linked entities. Through the forces generated by the platelet-platelet and platelet-wall links, aggregate growth can profoundly influence the flow, even to the extent that vessel closure can occur. Links may break if subject to sufficiently high stress by the fluid motion. As we discuss below, the models consist of stochastic and partial differential equations and auxiliary 'change of state' conditions. Model variables are fully coupled: the fluid carries the activator and platelets, while the interplatelet forces, potentiated by chemically-induced activation of the platelets, determine the local flow.

There are two major aspects of platelet behavior that the models attempt to 
capture. One is the mechanical interactions among the platelets, fluid, and vessel walls. The other is the platelet's detection and response to stimuli that can induce its activation. The IB approach is key to our modeling of the mechanical interactions. It also provides the scaffolding on which the platelet stimulus-response behaviors are modeled. We turn next to our use of the IB method in modeling the mechanical features of platelet aggregation.

\subsection{Mechanical interactions}

Our representation of discrete platelets as IB objects has evolved over the years: Initially, we treated platelets as point particles whose effective volume within the fluid was determined by the support of the approximate $\delta$-function and whose effective interaction distance with other platelets was determined by the range of an interplatelet repulsive force that was intended to prevent platelets from overlapping one another [15]. Currently, we model platelets as closed curves of interconnected IB points in 2D (see Fig. 2) and closed surfaces of interconnected IB points in 3D. A platelet's area or volume is determined by the region enclosed by the curve or surface, respectively, and is preserved because of the incompressibility of the fluid. There is no need for an explicit repulsive force, as the IB formulation automatically detects contact between the platelets and prevents one platelet from penetrating into the space occupied by another. This is an important advantage of the IB method over many other particle-tracking methods.

We describe the microscale model in its two-dimensional version for simplicity, and we describe the IB components of the model in discretized form, because that is how they are actually implemented and for some of these components, e.g., cohesive link formation, this is the natural way to describe them.

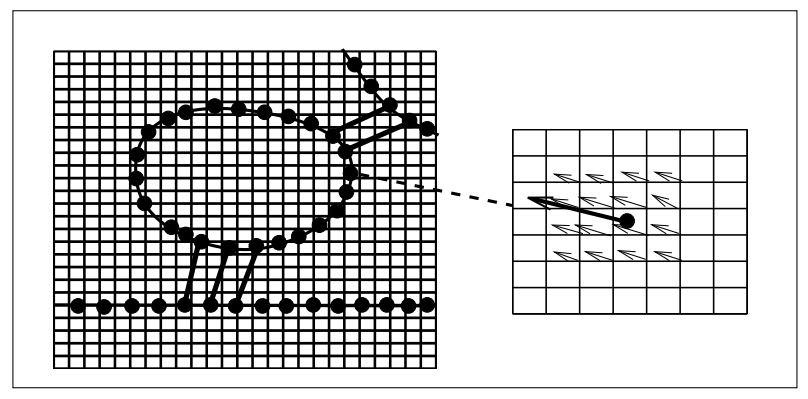

Fig. 2. Left: Schematic of discrete Cartesian grid for fluid and Lagrangian grids for platelets and vessel wall. Right: IB force is transmitted to a 4-by-4 portion of the grid.

In our microscale platelet aggregation calculations, the Navier-Stokes equations are discretized using finite difference methods on a simple uniform Cartesian grid. Hence, the fluid velocity, pressure, and force density are defined only 
at nodes $\mathbf{x}_{g}$ of this grid. Each platelet is represented by a ring of elasticallylinked Lagrangian IB points as shown schematically in Fig. 2. Let $\mathbf{X}_{p, i}$ denote the $i^{\text {th }}$ point of the ring that represents the $p^{\text {th }}$ platelet. We track the locations of these IB points for each platelet as time evolves. Between each pair of consecutive points $\mathbf{X}_{p, i}, \mathbf{X}_{p, i+1}$ on the platelet we assume there is a spring that generates forces on each of these points to try to maintain the separation between them at a prescribed value. (Arithmetic in the subscript $i$ is modulo the number of IB points in each platelet ring.) In addition, to give the platelet some rigidity, we assume that for each triplet of consecutive points $\mathbf{X}_{p, i-1}, \mathbf{X}_{p, i}, \mathbf{X}_{p, i+1}$, there is a 'hinge spring' that generates forces that try to maintain the angle formed by the vectors $\mathbf{X}_{p, i}-\mathbf{X}_{p, i-1}$ and $\mathbf{X}_{p, i+1}-\mathbf{X}_{p, i}$ at a prescribed value. (An alternative way to give the platelet rigidity, used in our 3D modeling, is to include an additional IB point initially at the platelet's center of mass and to add spring forces that try to maintain the distance between this point and each IB point on the platelet surface at a prescribed value.) The (linear) spring force on platelet point $\mathbf{X}_{p, i}$ due to connections with its neighbors in the ring is:

$$
S\left(\left\|\mathbf{X}_{p, i}-\mathbf{X}_{p, i+1}\right\|-r\right) \frac{\mathbf{X}_{p, i+1}-\mathbf{X}_{p, i}}{\left\|\mathbf{X}_{p, i+1}-\mathbf{X}_{p, i}\right\|}+S\left(\left\|\mathbf{X}_{p, i}-\mathbf{X}_{p, i-1}\right\|-r\right) \frac{\mathbf{X}_{p, i-1}-\mathbf{X}_{p, i}}{\left\|\mathbf{X}_{p, i-1}-\mathbf{X}_{p, i}\right\|}
$$

Suitable rearrangement of this expression shows that it is a discretization of the tension force expression $(\partial(T \boldsymbol{\tau}) / \partial q) d q$ discussed earlier in the case of a Hookean tension rule with stiffness $S$ and rest length $r$. The expression for the hinge-spring force is more complex (see [13].)

The walls of the blood vessel are also modeled as chains of elastically-linked IB points. In addition to the spring forces and hinge forces between neighboring wall points, each IB point $\mathbf{X}_{w, i}$ on the wall is connected by a spring to a corresponding 'tether point' $\mathbf{X}_{w, i}^{\text {tether }}$. For stationary vessel walls, the location of each tether point is held fixed in time. To model vasoconstriction or other vascular motions that can accompany vessel injury, the motion of the tether points would be a prescribed function of time or other model variables.

When a cohesive link connects IB points on two different platelets, there is an additional force on each of these points. Suppose link $l$ connects point $i_{1}(l)$ on platelet $p_{1}(l)$ to point $i_{2}(l)$ on platelet $p_{2}(l)$. Then, a force increment

$$
S^{\mathrm{coh}}\left(\left\|\mathbf{X}_{p_{2}(l), i_{2}(l)}-\mathbf{X}_{p_{1}(l), i_{1}(l)}\right\|-r^{\mathrm{coh}}\right) \frac{\mathbf{X}_{p_{2}(l), i_{2}(l)}-\mathbf{X}_{p_{1}(l), i_{1}(l)}}{\left\|\mathbf{X}_{p_{2}(l), i_{2}(l)}-\mathbf{X}_{p_{1}(l), i_{1}(l)}\right\|}
$$

is applied at point $\mathbf{X}_{p_{1}(l), i_{1}(l)}$ and the negative of this force increment is applied at point $\mathbf{X}_{p_{2}(l), i_{2}(l)}$. Here, $S^{\text {coh }}$ and $r^{\text {coh }}$ are the stiffness and rest length of cohesive links. A similar expression is used to calculate the forces generated by an adhesive link joining an IB point on a platelet to an IB point on a vessel wall. (Of course nonlinear or viscoelastic spring forces can be used for the intraplatelet springs and cohesive/adhesive links if desired.) 
The mechanical state of the model system at any time is described by the fluid velocity and the locations of all of the IB points, as well the configuration of elastic links which join IB points to one another and the properties of these links. Here we summarize how the fluid velocity and IB point locations are advanced during one timestep of a simulation. Below we describe how other aspects of the model system (activator chemical distribution, platelet activation status, configuration of cohesive and adhesive links) are updated.

There are four steps to updating the velocity and IB point locations. First, the resultant $\mathbf{F}_{q}$ of all of the IB force contributions that act on the $q^{\text {th }}$ IB point is calculated for each $q$. Next, these forces are distributed to the Eulerian grid used for the fluid dynamics equations using a discrete version of Eq.(3):

$$
\mathbf{f}\left(\mathbf{x}_{g}\right)=\sum_{q} \mathbf{F}_{q} \delta_{h}\left(\mathbf{x}_{g}-\mathbf{X}_{q}\right) d q
$$

Here, $\mathbf{x}_{g}$ and $\mathbf{X}_{q}$ are the coordinates of grid point $g$ and IB point $q$, respectively, $\mathbf{F}_{q}$ is the IB force (per unit $q$ ) on this point, $d q$ is the increment in parameter $q$ between consecutive discrete IB points, and $\delta_{h}$ is a discrete approximation to a two-dimensional $\delta$-function. With the fluid force density $\mathbf{f}_{g}$ now known at each grid point, the fluid velocity is updated taking one step with a discrete Navier-Stokes solver. We use a projection-method type of Navier-Stokes solver [17]. Denoting the new velocity field by $\mathbf{u}_{g}^{\text {new }}$, the fourth step of the update process is described by a discrete analog of Eq.(4)

$$
\mathbf{X}_{q}^{\text {new }}=\mathbf{X}_{q}+d t \mathbf{U}_{q} \equiv \mathbf{X}_{q}+d t \sum_{g} \mathbf{u}_{g}^{\text {new }} \delta_{h}\left(\mathbf{x}_{g}-\mathbf{X}_{q}\right) h^{2}
$$

where $h$ is the fluid grid spacing and $\delta_{h}$ is the same approximate $\delta$-function as used in Eq.(8). (Below we discuss an additional term in the update equation for $\mathbf{X}_{q}$ that may used to model the effect of red blood cells on platelet motion.)

The function $\delta_{h}$ is chosen to be a tensor product of one-dimensional approximate $\delta$-functions,

$$
\delta_{h}(\mathbf{x}) \equiv \frac{1}{h^{2}} \phi\left(\frac{x}{h}\right) \phi\left(\frac{y}{h}\right)
$$

where $\mathbf{x}=(x, y)$. A common choice for the function $\phi(r)$ is

$$
\phi(r)= \begin{cases}\frac{1}{4}\left(1+\cos \left(\frac{\pi r}{2}\right)\right), & -2 \leq r \leq 2 \\ 0, & \text { otherwise }\end{cases}
$$

which ensures that the entire IB force is transmitted to the grid, that the force density on the grid is a continuous function of the IB point locations, and that the communication between grid and IB points is very localized. In fact, the IB force $\mathbf{F}_{q}$ is spread to a 4 -by-4 region of the grid surrounding IB point $\mathbf{X}_{q}$, and the velocity of this IB point is interpolated from the velocity values at the same grid points (see Fig. 2). 
Note that two IB objects can come arbitrarily close to one another. If two IB points are separated by a distance less than $4 h$ (the width of the approximate $\delta$-function), there is overlap in the grid velocities from which the IB point velocities are interpolated using Eq.(9). As the distance between the points approaches 0 , their velocities approach a common value. This implies that IB objects cannot interpenetrate so there is no need for extra forces or rules to prevent overlap.

\subsection{Red-blood-cell-induced platelet motion}

As noted in Section 3, tumbling and colliding red blood cells impart to the platelets a 'random' motion. In principle, we could use the IB method to model each red blood cell and its motion and the consequent effects on platelet motion would presumably emerge from the simulations. Unfortunately, it is currently not practical to track hundreds of red blood cells over the time periods (tens of seconds) over which thrombi form in small vessels. Bagchi [18] recently used the IB method to simulate the motion of hundreds of red blood cells but only for very short periods $\left(<\frac{1}{2} \mathrm{sec}\right)$. Since direct simulation of red blood cells is not yet feasible in our simulations, we instead model their effects on platelet motion, guided by empirical observations, without explicitly treating the red blood cells in detail.

The effect of red blood cells on platelet motion is often described as an effective 'diffusion'. The simplest way of incorporating this into the microscale models is to add a random step to each platelet's motion during each time step. This is accomplished by specifying that the motion of platelet IB points satisfy the equation,

$$
d \mathbf{X}_{p, i}(t)=\mathbf{U}_{p, i}(t) d t+d \mathbf{B}_{p}
$$

where $d \mathbf{B}_{p}=(2 D d t)^{1 / 2} \mathbf{R}$ with $\mathbf{R}$ a Gaussian random variable with mean 0 and variance 1 , and $\mathbf{U}_{p, i}(t)$ is the interpolated velocity given in Eq.(9). The effective diffusion coefficient $D$ may be allowed to depend on the local shear rate and on the prescribed red blood cell volume fraction as suggested by experiments $[11,12]$. The random step is taken only by individual platelets, not by aggregated ones, because the effect of the red blood cells should be less for platelets bound together in an aggregate.

An alternative way to incorporate the effect of the red blood cells is, for each platelet $p$, to add a random force $\mathbf{F}_{p}^{\text {random }}$ to the IB force at each point on platelet $p$ before its IB forces are transmitted to the surrounding fluid. The random force thus contributes to the local force density on the fluid. In this case the platelet motion is by advection in the resulting velocity field, without the additional random step $\left(d \mathbf{B}_{p}\right)$. In this approach, the platelets and the surrounding fluid are both affected by the random forces and there is no relative 
motion between the platelets and the fluid. We are currently experimenting with this approach, considering different ways of choosing random force vectors and distributing the forces among the IB points of the platelets, in order to obtain platelet motions consistent with empirical observations.

\subsection{Activator transport and secretion}

When a platelet becomes activated, it begins to secrete activator chemical (e.g., ADP or thrombin) into the fluid, and so the platelet serves as a (moving) source of activator. Once the chemical has been released into the fluid, we assume it moves by advection with the fluid and diffusion relative to the fluid. We also allow for the possibility that the activator is degraded in the fluid. The equation describing the evolution of the activator concentration $c(\mathbf{x}, t)$ is therefore

$$
c_{t}+\mathbf{u} \cdot \nabla c=D_{\mathrm{c}} \Delta c+s(\mathbf{x}, t)-K c
$$

where $D_{\mathrm{c}}$ is the activator diffusion coefficient, $K$ is its degradation rate, and $s(\mathbf{x}, t)$ is the source of chemical due to secretion by platelets. The source function is defined by the sum,

$$
s(\mathbf{x}, t)=\sum_{k} H\left(\mathbf{x}, \mathbf{X}_{k, 1}(t), \mathbf{X}_{k, 2}(t), \ldots\right) G\left(t-t_{k}\right) .
$$

Here, $k$ ranges over the indices of activated platelets in the domain, $t_{k}$ is the time of activation of platelet $k, G$ describes the (prescribed) timecourse of activator secretion following platelet activation, and $H$ describes the spatial distribution of the source due to platelet $k$. For example, in a variant of the model in which the activator diffuses both within the platelets and in the surrounding fluid, the secreted activator can be distributed uniformly within the region bounded by the IB curve that represents the platelet. The secreted chemical then gradually diffuses across the platelet boundary into the fluid (see Fig. 4).

In reality, the activator chemical should move only in the portions of the domain occupied by fluid, not those occupied by platelets. That is, activator should be able to diffuse up to but not across platelet walls. The same is true with regard to the vessel walls. For straight vessel walls, it is simple to impose the no flux condition on the activator chemical within the context of a finitedifference approximation to Eq.(13). For irregular vessel walls and for moving platelets, it is more difficult. One approach that we have used for irregular vessel walls (see Fig. 8 below) is to use the Immersed Interface method (IIM) which is a method for modifying the finite difference stencils near a boundary to impose boundary or jump conditions [19-21]. This works well for stationary irregular boundaries, but since there is significant overhead in determining the modified stencils, it is less well suited to moving boundaries. Another approach 
[22] with which we are currently experimenting is to define a potential field $\Phi$ with respect to the current platelet boundaries and to update the chemical concentration by taking a step of a discretized version of the modified transport equation

$$
c_{t}+\mathbf{u} \cdot \nabla c=-\nabla \cdot\left(-D_{\mathrm{c}} \nabla c-c \nabla \Phi\right)+s(\mathbf{x}, t)-K c
$$

Since the additional term describes a flux of chemical down gradients in $\Phi$, we can design $\Phi$ to be a barrier to chemical crossing the platelet boundary from the fluid. In fact, $\Phi$ can be defined by using the discrete $\delta$-function $\delta_{h}$ to spread to the computational grid an appropriate scalar function defined at the IB points which make up each platelet's boundary. Tests of the potential barrier approach are very encouraging and will be reported on elsewhere.

\subsection{Modeling activation, adhesion, and cohesion}

Our modeling of other aspects of platelet behavior, including activation, adhesion, and cohesion, is faciliated by our Lagrangian representation of platelet's surface. Recall that activation of a platelet can be stimulated by a platelet's interaction with specific molecules exposed on the damaged vascular wall or present in the blood plasma, and that platelet binding to the injured wall or to another platelet is accomplished by the formation of molecular bonds involving specific molecules on the respective surfaces and in the blood plasma.

Until recently, our modeling of these events has been relatively simplistic, and while this has allowed us to make qualitative comparisons between simulation results and real aggregation, it precludes meaningful quantitative comparisons. New biological information allows us to begin to make more sophisticated models and to begin to make quantitative comparisons between simulated and real aggregation events. It is a strength of our computational approaches that they facilitate incorporating into the models much more sophisticated treatment of platelet responses to stimuli and of adhesion and cohesion.

For the simulations shown in Figs.(3-4), we made the following assumptions about platelet adhesion to the damaged wall: i) Whenever a platelet comes within a prescribed distance of the injury, it immediately adheres. ii) Adhesion is accomplished by formation of an elastic link between an IB point on the platelet and a nearby IB point on the vessel wall. iii) Additional links form quickly as long as there are pairs of unbound IB points on the platelet and vessel wall that are sufficiently close to one another. iv) Each link behaves like a Hookean spring with a stiffness coefficient that is constant in time. v) Link formation is irreversible except that a link breaks if subject to a sufficiently large force. Similar assumptions governed our modeling of cohesion between activated platelets. 
We also assumed that activation is an immediate response to a platelet's contact with the injured wall or its exposure to a sufficiently high concentration of activator, and that activation i) instantaneously allows the platelet to cohere to other (nearby) activated platelets and ii) instantaneously causes activator release into the surrounding fluid. As with adhesion, whenever an unactivated platelet came within a prescribed distance of the injury, it was activated. To respond to the chemical activator, unactivated platelets must sense the level of activator in their vicinity, and this was done by interpolating, for each unactivated platelet, the activator concentration from the grid to each of its IB points. If the average of these interpolated concentrations was above a prescribed threshold level, the platelet became activated.

Each platelet's activation status was tracked with a simple 'activation flag' with values true and false. We tracked each cohesion and adhesion link by maintaining lists from which we could extract, for any link $l$, the indices of the IB objects (platelets or walls) and of the IB points within those objects joined by link $l$, as well as the resting length and stiffness of that link. From this information we could access the coordinates of the points joined by the link and calculate the force generated by the link (see Eq.(7)). This force (with appropriate sign) was added to the IB force at each of the two IB points joined by the link. When a new link formed, the relevant information was added to these lists; when an existing link broke, the corresponding information was removed.

In recent years, it has become clear that a real platelet's surface is studded with large numbers of molecules that perform different (but in some cases overlapping) roles in the platelet's detection and response to vascular injury. These include approximately 25,000 GPIb receptors that can bind to vWF molecules adsorbed onto the exposed subendothelial matrix [23], large numbers of receptors (GP-VI and $\alpha_{2} \beta_{1}$ ) that bind to subendothelial collagen [24], about 50,000 $\alpha_{I I b} \beta_{3}$ receptors that are involved both in platelet-platelet and platelet-wall binding [25], and at least two different types of receptors for each of the activators ADP and thrombin $[26,27]$. Within the framework of our existing Lagrangian representation of each platelet, we can easily represent each class of these receptors and include their associated reactions in the model.

To do this we partition the surface of a model platelet into small patches around each of its IB points and we assign to each IB point an appropriate fraction of each type of receptor on the platelet surface. As simulation events unfold, ligands bind to and unbind from these receptors, and we track these events by associating with each IB point a vector whose components are the number densities of bound and unbound receptors of each type at that IB point. Using appropriate kinetic equations that 'live' at the IB point, we follow the formation/breaking of new bonds, the activation of receptors, and the overall stimulus for activation to which the platelet is exposed. For example, for 
adhesion, link formation occurs, as in the current model, when an IB point on a platelet comes sufficiently close to an IB point on the injured vessel wall, and the link will, as now, represent a collection of molecular bonds. However, rather than treating the link's stiffness as constant, which is tantamount to assuming that all of these bonds form simultaneously, we will follow the dynamics of bond formation and breakage and relate the mechanical properties of the link at each instant of time to the number and type of each bond present then. Let $n_{j}(t)$ denote the number density of bonds of type $j$ between a particular pair of linked IB points. These bonds contribute an amount $S d a n_{j}(t)\left(l(t)-l_{0}\right)$, to the magnitude of the force that the link exerts on each of the two IB points. Here, $l(t)$ is the distance between the two IB points, $l_{0}$ is its resting length, $S$ is the stiffness of an individual bond, and $d a$ is the area of the surface patch associated with the IB point. The sum of these contributions is transmitted to the fluid grid near the corresponding linked IB points.

The evolution of the bond density $n_{j}(t)$ will be governed by an equation of the form:

$$
\frac{d n_{j}}{d t}=k_{j}^{+}\left(n_{j}^{\max }-n_{j}\right)-k_{j}^{-}\left(\frac{f}{\sum_{k} n_{k}}\right) n_{j}
$$

Here, $f$ is the force on the link that results from flow-mediated stretching, and which we assume is distributed equally to all the $\sum_{k} n_{k}$ bonds that make up this link. In this equation, we assume that the bond dissociation rate $k_{j}^{-}$is an increasing function of the force on the bond as is typical for molecular bonds [28]. For some types of bonds, the maximum number of bonds possible $\left(n_{j}^{\max }\right)$ may vary with the platelet's activation status [29]. Cohesive links between activated platelets will be treated in a simular fashion, but modified to take into account the fact that the bonds between platelets are formed by a plasma protein binding to sites on each of the two platelets.

A platelet's interaction with activator chemical in the plasma can be handled using similar ideas. Let $r$ denote the number density of receptors associated with a particular IB point and let $b$ be the number density of receptors occupied by an activator molecule. Then $b$ evolves according to an equation of the form

$$
\frac{d b}{d t}=k_{\mathrm{on}} c(\mathbf{X})(r-b)-k_{\mathrm{off}} b
$$

where $c(\mathbf{X})$ is the concentration of activator at the IB point's location as interpolated from nearby grid points, and $k_{\text {on }}$ and $k_{\text {off }}$ are binding constants. When we incorporate the coagulation enzyme network into our platelet aggregation models, a similar approach will be used to track the interactions between coagulation molecules in the plasma and binding sites on the platelet's surface. Additional reactions between two platelet-surface-bound species will involve ordinary differential equations relating their surface densities. 


\subsection{Microscale simulation results}

Fig. 3 shows snapshots of a portion of the computational domain during a microscale aggregation simulation using the two-dimensional model. Recent theoretical [30-32] and experimental studies [33] highlight the fluid dynamic importance of the resting platelet's discoid shape and this is straightforward to take into account in the IB model. In our simulations, each platelet is initially represented by an approximately rigid ellipse comprised of twentyeight sequentially linked IB points. The platelet shape is maintained using stiff hinge forces as described in Section (4.1). Upon a platelet's activation, the hinge force parameters for that platelet are reset so that the platelet's target shape is circular and the platelet is more deformable. This allows the platelet to change shape, as do real platelets upon activation and adhesion to the vessel wall or to a developing thrombus [24]. In the simulation, a portion of the bottom vessel wall is designated as injured and platelets that contact it, adhere to it and become activated. Only the portion of the computational domain near the injured wall is shown in Fig. 3.

Each of the two small aggregates that form early in the simulation disturb the flow to a small extent. Although the aggregate in the center of the injured region is initially larger, the aggregate at the upstream end of the injury grows more quickly and partially sheilds the downstream portions of the injured wall, slowing growth of the centrally-located aggregate. Together these aggregates disturb the flow sufficiently that few platelets contact and adhere to the downstream portion of the injured wall. One can see linear chains of platelets bend in response to the fluid forces, bringing platelets of the two aggregates into close proximity and potentially leading to consolidation of the adherent platelets into one larger thrombus. For an aggregate that projects substantially into the vessel lumen there is a substantial strain on its most upstream attachments to the vessel wall (see upstream end of last panel) and this can lead to breaking of these attachments allowing the aggregate to roll downstream. For this simulation, no activator chemical was included; unactivated platelets became activated if they came sufficiently close to another activated platelet. This simulation pertains to events in a $200 \mu \mathrm{m}$ long segment of a $37 \mu \mathrm{m}$ diameter arteriole in which the peak inflow velocity was $1.0 \mathrm{~cm} / \mathrm{sec}$. The aggregates developed during a time period of approximately 2.5 sec. This is one to two orders of magnitude faster than thrombi develop in injured arterioles [34]. The speedup here is due to the very simple rules used for platelet adhesion and cohesion and for platelet activation (see Sec.4.4), and to our use of a platelet concentration somewhat higher than physiological.

Fig. 4 shows snapshots from a brief three-dimensional simulation [16]. For this simulation, a platelet is represented by a triangulated surface mesh of elastically-connected IB points. The surface mesh was constructed beginning 


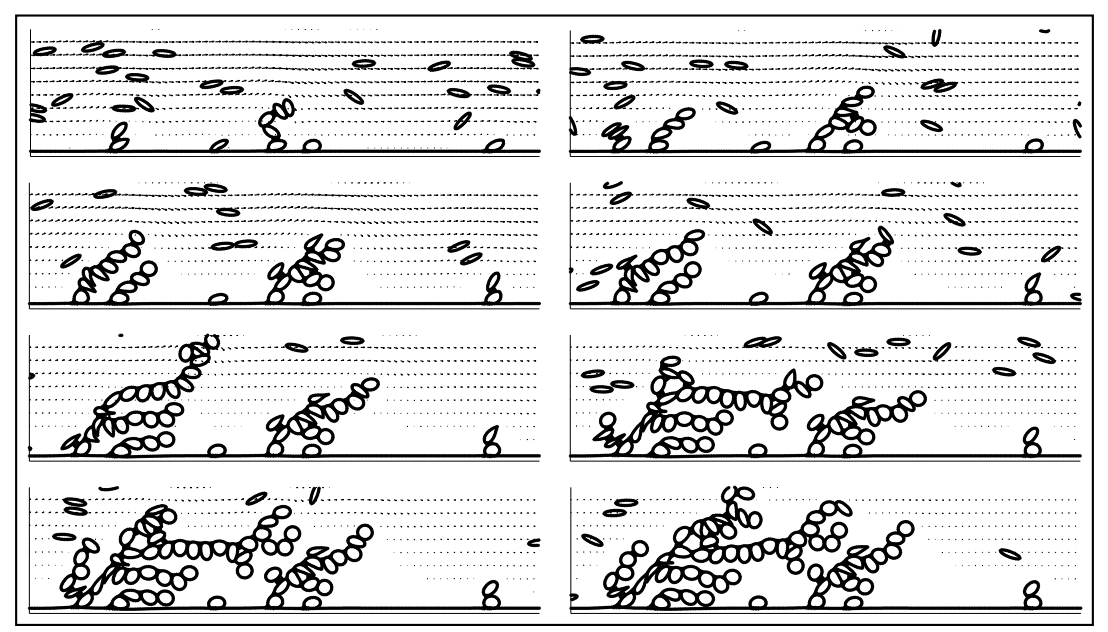

Fig. 3. Development of platelet aggregates during a 2D microscale model simulation. Rings depict immersed boundary platelets, arrows show velocity field. A portion of the computational domain is shown. Time advances left to right and top to bottom.

with the vertices of a truncated icosahedron ('soccerball'), introducing new vertices to triangularize each hexagonal and pentagonal face, and then refining each of the resulting triangles into four smaller triangles. Platelet contact with the injured portion of the vessel wall leads to activation of the platelet and its secretion of activating chemical into the blood plasma. The isosurface bounds the region in which the activator concentration is sufficiently high to activate platelets. Below it, we see the development of a small wall-bound aggregate and a number of platelet doublets not attached to the vessel wall.

\section{Continuum Models of Platelet Thrombosis}

Our continuum models of platelet aggregation are intended to describe events in large diameter blood vessels such as the coronary arteries in which it is infeasible to track all platelets and all adhesive and cohesive links individually. These models are based on the same interactions as the microscale models but follow the evolution of density functions that describe the distribution of nonactivated and activated platelets and of cohesive and adhesive links.

As indicated in Fig. 5, two spatial scales arise in this problem. One, the macroscale, is the scale of the vessel (1-2 millimeters) which also is the scale of clots that grow to substantially or completely occlude the vessel. The other, the microscale, is that of platelets (1-2 microns). As a consequence two sets of spatial variables appear in the models. The vector $\mathbf{x}$ refers to the macroscale and the statement $\|\mathbf{x}\|=O(1)$ signifies distances on the order of a millimeter. The vector $\mathbf{y}$ refers to the microscale and $\|\mathbf{y}\|=O(1)$ means distances on the order of a micron. The ratio of the platelet scale to the vessel scale is denoted 

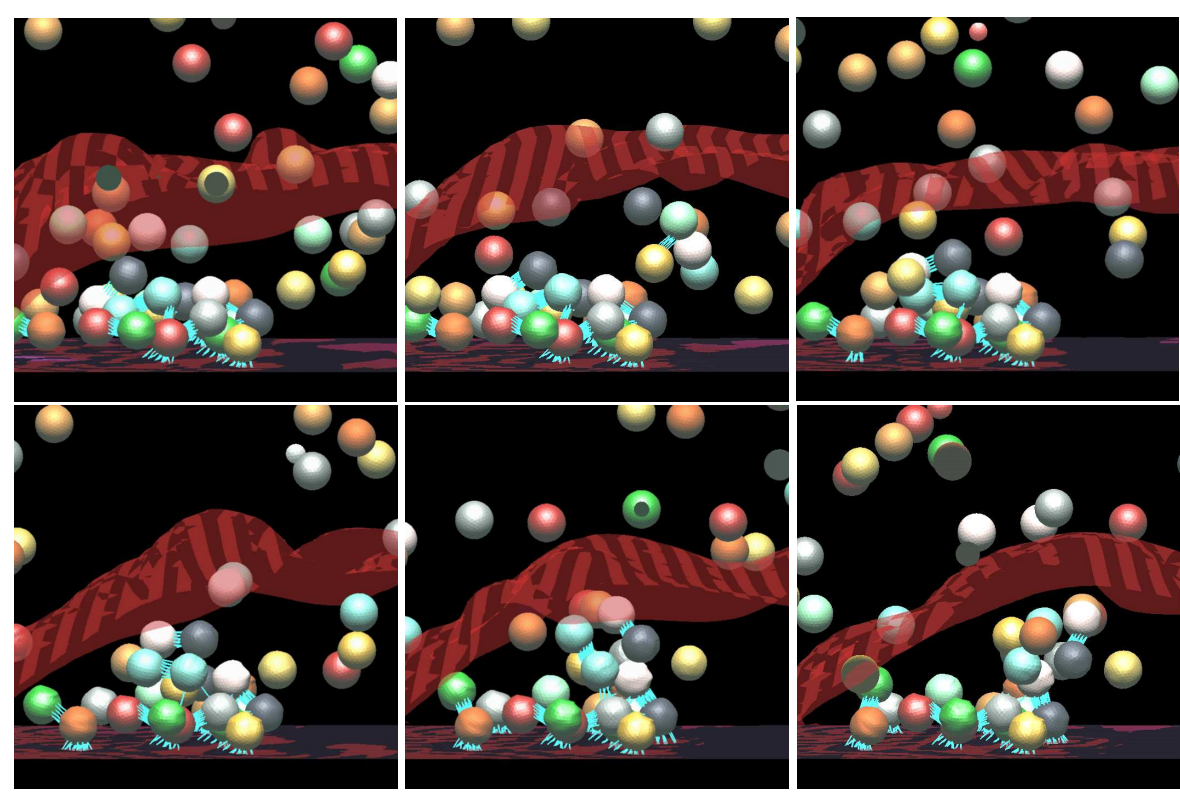

Fig. 4. Close up of formation of a small aggregate during a 3D microscale simulation. Each "ball" is actually an IB representation of a platelet using a triangulated surface mesh of 362 elastically connected points. (The colors of the platelets are not meaningful.) Line segments show cohesive and adhesive links. Below the red isosurface the activator concentration is sufficiently high to activate platelets. See [16] for more information.

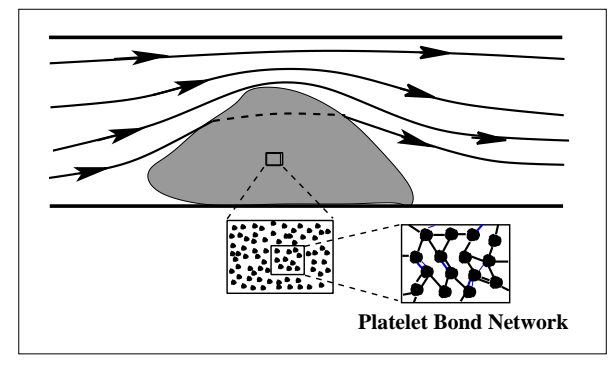

Fig. 5. The continuum models involve two spatial scales; the vessel scale is on the order of millimeters, and the platelet-platelet cohesion scale is on the order of microns.

by $\epsilon \ll 1$, and the model's equations are actually the leading order terms in expansions in $\epsilon$.

\subsection{Fluid-phase multiscale model}

We begin with a description of the models in the absence of vessel walls, so that all interactions are between fluid-phase species. (Below we show how interactions with the vessel walls are added to the models.) The unknowns in the models are the fluid velocity $\mathbf{u}(\mathbf{x}, t)$ and pressure $p(\mathbf{x}, t)$, the concentrations of nonactivated and activated platelets, $\phi_{n}(\mathbf{x}, t)$ and $\phi_{a}(\mathbf{x}, t)$ respectively, the 
concentration of activator chemical $c(\mathbf{x}, t)$, and a function $E(\mathbf{x}, \mathbf{y}, t)$ that describes the distribution of interplatelet cohesive links. We refer to this function as the 'elastic link function'. $E$ is defined so that $E(\mathbf{x}, \mathbf{y}, t) d \mathbf{x} d \mathbf{y}$ is the number of elastic links which connect activated platelets at location $\mathbf{x}$ to activated platelets a short distance away at $\mathbf{x}+\epsilon \mathbf{y}$; hence, $E$ has dimensions of number of links per volume per volume. From the distribution of elastic links at any time $t$, we can calculate the stresses that the links exert on the fluid.

The equations for the solution-phase model fall into three groups. The first group consists of the Navier-Stokes equations for the fluid motion.

$$
\rho\left(\mathbf{u}_{t}+\mathbf{u} \cdot \nabla \mathbf{u}\right)=-\nabla p+\mu \Delta \mathbf{u}+\nabla \cdot \underline{\underline{\sigma}}^{\mathrm{p}}, \quad \nabla \cdot \mathbf{u}=0
$$

These equations contain a forcing term $\nabla \cdot \underline{\underline{\sigma}}^{\mathrm{p}}$ which involves the "cohesive stress tensor' $\underline{\underline{\sigma}}^{\mathrm{p}}$ that is generated by the interplatelet elastic links as we describe below.

The second group of equations governs the transport of platelets and activator chemical:

$$
\begin{aligned}
& \left(\phi_{n}\right)_{t}+\mathbf{u} \cdot \nabla \phi_{n}=D_{n} \Delta-R(c) \phi_{n} \\
& \left(\phi_{a}\right)_{t}+\mathbf{u} \cdot \nabla \phi_{a}=\quad R(c) \phi_{n} \\
& c_{t}+\mathbf{u} \cdot \nabla c=D_{c} \Delta c+A R(c) \phi_{n}-K c
\end{aligned}
$$

Eq.(19) embodies the assumptions that nonactivated platelets move by advection and diffusion, and that they disappear, through activation, at a rate that depends on the local concentration of activator chemical. The diffusive term in this equation is intended to model the effect of red blood cells on platelet motion. According to Eq.(20), activated platelets move by advection and appear due to activation of nonactivated platelets. No diffusive term appears here, because, as in the microscale models, we assume that the effect of red blood cells on aggregated platelets is small. Eq.(21) indicates that activator chemical moves by advection and diffusion, and that it is produced at each location at a rate proportional to the rate of platelet activation there.

The third group of equations pertains to the interplatelet links. The equation

$$
E_{t}+\mathbf{u} \cdot \nabla_{x} E+(\mathbf{y} \cdot \nabla \mathbf{u}) \cdot \nabla_{y} E=\alpha(\|\mathbf{y}\|) \phi_{a}^{2}-\beta(\|\mathbf{y}\|) E
$$

describes the evolution of the elastic link function by advection in $\mathbf{x}$ at velocity $\mathbf{u}$, advection in $\mathbf{y}$ at velocity $\mathbf{y} \cdot \nabla \mathbf{u}$, formation of new links at rate $\alpha(\|\mathbf{y}\|) \phi_{a}^{2}$, and breaking of existing links at rate $\beta(\|\mathbf{y}\|) E$. The unusual advection term $(\mathbf{y} \cdot \nabla \mathbf{u}) \cdot \nabla_{y} E$ arises because of the slight difference in velocity at the two ends of a link. The link formation and breaking rate functions, $\alpha(\|\mathbf{y}\|)$ and $\beta(\|\mathbf{y}\|)$ are assumed to depend on the distance $\|\mathbf{y}\|$ between the linked platelets. Link formation occurs at a rate proportional to $\phi_{a}^{2}$ because each link joins two activated platelets, and is isotropic (in $\mathbf{y}$ ) because $\alpha$ depends only on the length 
of $\mathbf{y}$. In a shear flow, the advection term $(\mathbf{y} \cdot \nabla \mathbf{u}) \cdot \nabla_{y} E$ has the important effect of progressively stretching the links and aligning them with the flow. The equation

$$
\underline{\underline{\sigma}}^{\mathrm{p}}(\mathbf{x}, t)=\int_{\mathbf{y}} E(\mathbf{x}, \mathbf{y}, t)\left\{\frac{1}{2} S(\|\mathbf{y}\|) \mathbf{y} \mathbf{y}^{T}\right\} d \mathbf{y}
$$

shows how the cohesive stress tensor is determined from the distribution of elastic links. In Eq.(23), $S(\|\mathbf{y}\|)$ denotes the stiffness of a single link of length $\|\mathbf{y}\|$. The formula for $\underline{\underline{\sigma}}^{\mathrm{p}}$ can be derived by summing up the individual contributions to the stress at $\mathbf{x}$ of each link which joins a platelet at $\mathbf{x}$ to a platelet elsewhere.

The model, although formulated completely in terms of Eulerian variables, is similar to the IB method in a very important way. As platelet aggregates grow in the model, the fluid is affected solely through the forcing term $\nabla \cdot \underline{\underline{\sigma}}^{\mathrm{p}}$ in Eq.(18); there is no change in geometry as a result of aggregate growth.

It is useful to introduce an additional variable $z^{\mathrm{p}}(\mathbf{x}, t)=\int_{\mathbf{y}} E(\mathbf{x}, \mathbf{y}, t) d \mathbf{y}$ which measures the concentration of elastic links emanating from activated platelets at $\mathbf{x}$. Since the presence of an aggregate is manifest only through the stresses from these links, we regard $z^{\mathrm{p}}$ as a useful indicator of the extent of aggregation at point $\mathbf{x}$, and refer to $z^{\mathrm{p}}$ as the 'aggregation intensity'. For now, $z^{\mathrm{p}}$ is just a diagnostic variable that allows us to monitor where aggregation has occured, but later it will enter into an approximate form of the model.

\subsection{Model reductions}

Through the function $E(\mathbf{x}, \mathbf{y}, t)$, the model just presented describes both microscale and macroscale events. E impacts the rest of the model only through the stress tensor $\underline{\underline{\sigma}}^{\mathrm{p}}$, and this stress tensor and all other model variables depend only on the macroscale spatial variables $\mathbf{x}$. This prompts the question of whether an evolution equation for $\underline{\underline{\sigma}}^{\mathrm{p}}$, which involves only the macroscale variable $\mathbf{x}$, can be derived. Toward this end, multiply Eq.(22) by $\left\{\frac{1}{2} S(\|\mathbf{y}\|) \mathbf{y} \mathbf{y}^{T}\right\}$ and integrate over the microscale variable $\mathbf{y}$ to obtain:

$$
\begin{aligned}
\underline{\underline{\sigma}}_{t}^{\mathrm{p}}+\mathbf{u} \cdot \nabla \underline{\underline{\sigma}}^{\mathrm{p}} & =\underline{\underline{\sigma}} \underline{\underline{\nabla \mathbf{u}}}+\left(\underline{\underline{\sigma}}^{\mathrm{p}} \underline{\underline{\nabla \mathbf{u}}}\right)^{T}+\alpha_{2} \phi_{a}^{2} \underline{\underline{I}} \\
& -\int \beta(\|\mathbf{y}\|) E\left\{\frac{1}{2} S(\|\mathbf{y}\|) \mathbf{y} \mathbf{y}^{T}\right\} d \mathbf{y} \\
& +\int\left(\mathbf{y}^{T} \underline{\underline{\nabla \mathbf{u}}} \mathbf{y}\right)\left\{\frac{1}{2} S^{\prime}(\|\mathbf{y}\|) /\|\mathbf{y}\|\right\} E \mathbf{y y}^{T} d \mathbf{y}
\end{aligned}
$$


where $\alpha_{2}=\int_{\mathbf{y}}\left\{\frac{1}{2} \alpha(\|\mathbf{y}\|) S(\|\mathbf{y}\|)\|\mathbf{y}\|^{2}\right\} d \mathbf{y}$ is a constant that reflects the rate of stress-production due to new link formation. The first five terms in Eq.(25) involve only the macroscale variable $\mathbf{x}$. The last two terms are problematic in general; they cannot be expressed in terms of the existing model variables that depend only on the macroscale variable $\mathbf{x}$.

If we impose two restrictions on the model, namely, that the links behave as linear springs so $S(\|\mathbf{y}\|)=S_{0}$ and $S^{\prime}(\|\mathbf{y}\|)=0$, and that links break at a constant rate independent of how stretched they are so $\beta(\|\mathbf{y}\|)=\beta_{0}$, then the two problematic terms vanish, and we have an exact evolution equation for $\underline{\underline{\sigma}}^{\mathrm{p}}$.

$$
\underline{\underline{\sigma}}_{t}^{\mathrm{p}}+\mathbf{u} \cdot \nabla \underline{\underline{\sigma}}^{\mathrm{p}}=\underline{\underline{\sigma}}^{\mathrm{p}} \underline{\underline{\nabla \mathbf{u}}}+\left(\underline{\underline{\sigma}}^{\mathrm{p}} \underline{\underline{\nabla \mathbf{u}}}\right)^{T}+\alpha_{2} \phi_{a}^{2} \underline{\underline{I}}-\beta_{0} \underline{\underline{\sigma}}^{\mathrm{p}}
$$

Under the same restrictions, the aggregation intensity $z^{\mathrm{p}}$ satisfies the evolution equation:

$$
z_{t}^{\mathrm{p}}+\mathbf{u} \cdot \nabla z^{\mathrm{p}}=\alpha_{0} \phi_{a}^{2}-\beta_{0} z^{\mathrm{p}}
$$

where $\alpha_{0}=\int_{\mathbf{y}} \alpha(\|\mathbf{y}\|) d \mathbf{y}$. The set of equations Eqs.(18)-(21) and Eq.(25) form a closed system of equations that govern the behavior of the model under the restrictions that $S=S_{0}$ and $\beta=\beta_{0}$ are constant. We refer to these equations as the 'special' form of the model. We studied its properties $[35,36]$, and among other things, saw that it can demonstrate a phase transition that we interpret as platelet aggregation. Consider a period box in which initially $\phi_{n}=1$ and $\phi_{a}=0$ for all $\mathbf{x}$. Suppose that a background force is applied to the fluid to drive a periodic stagnation-point flow as shown in Fig. 6a, and that at $t=0$ a sufficiently high concentration of activator is added in a region centered at the stagnation point. Early on, activation occurs, links form, and this early aggregate is stretched by the elongational flow along the $x$-direction. As time progresses, further activation and net link formation occur and the link distribution becomes more and more aligned with the flow and therefore able to generate forces to resist further elongation. By the end of the simulation, the core of the aggregate has solidified and the flow within it has dropped essentially to zero.

The special form of the model is limited by the restrictions imposed in its derivation. In particular, the requirement that links break at a rate that is independent of stretch leads to nonphysical behaviors and makes it impossible to cause an aggregate to break up by subjecting it to force. Since real clots do break up (embolize), presumably because of the stresses the flowing blood applies to them, this is an unacceptable limitation. The full multiscale version of the model does allow the link breaking rate to depend on stretch, but using it means having to contend with the substantial computational expense of dealing with two sets of spatial variables. Referring to Eq.(25), recall that the reason we cannot in general get a macroscale equation for $\underline{\underline{\sigma}}^{\mathrm{p}}$ is that $\beta(\|\mathbf{y}\|)$ cannot be brought outside of the integral in the next to last term in this equation. (We are content for now with the restriction that the stiffness $S(\|\mathbf{y}\|)$ 

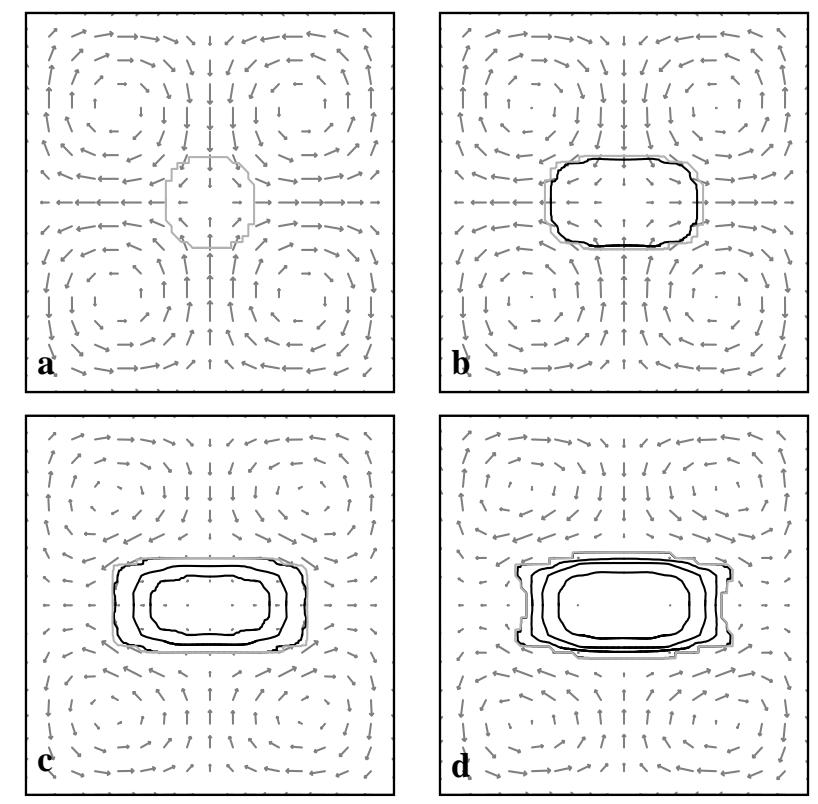

Fig. 6. (a-d) Snapshots at increasing times of the velocity field, an outer contour that encloses the region in which $c$ is sufficiently high to induce platelet activation, and inner contours that show levels of the aggregation intensity $z^{\mathrm{p}}$ (highest near the aggregate's center).

be constant, so the final term in Eq.(25) vanishes.) If $\beta$ were not a function of $\|\mathbf{y}\|$ itself, but of some macroscale quantity that reasonably represents $\|\mathbf{y}\|$, then $\beta$ could be brought outside of the integral and this term would reduce to $\beta \underline{\underline{\sigma}}^{\mathrm{p}}$. Consider the macroscale quantity $\operatorname{Tr}\left(\underline{\underline{\sigma}}^{\mathrm{p}}\right) / z^{\mathrm{p}}$, where $\operatorname{Tr}\left(\underline{\underline{\sigma}}^{\mathrm{p}}\right)$ denotes the trace of the tensor $\underline{\underline{\sigma}}^{\mathrm{p}}$. Recalling the definitions of $\underline{\underline{\sigma}}^{\mathrm{p}}$ and $z^{\mathrm{p}}$ we see that

$$
\frac{\operatorname{Tr}\left(\underline{\underline{\sigma}}^{\mathrm{p}}\right)}{z^{\mathrm{p}}}=\frac{S_{0}}{2}\left\{\frac{\int E(\mathbf{x}, \mathbf{y}, t)\|\mathbf{y}\|^{2} d \mathbf{y}}{\int E(\mathbf{x}, \mathbf{y}, t) d \mathbf{y}}\right\}=\frac{S_{0}}{2}\left\langle\|\mathbf{y}\|^{2}\right\rangle(\mathbf{x}, t),
$$

that is, $\operatorname{Tr}\left(\underline{\underline{\sigma}}^{\mathrm{p}}\right) / z^{\mathrm{p}}$ is a constant multiple of the mean-squared length of links emanating from platelets at $\mathbf{x}$. Its squareroot therefore serves as a reasonable surrogate for the actual link length, and so we make the approximation that $\beta$ is a function of this macroscale quantity rather than of $\|\mathbf{y}\|$. With this closure approximation, the equation for $\underline{\underline{\sigma}}^{\mathrm{p}}$ is

$$
\underline{\underline{\sigma}}_{t}^{\mathrm{p}}+\mathbf{u} \cdot \nabla \underline{\underline{\sigma}}^{\mathrm{p}}=\underline{\underline{\sigma}}^{\mathrm{p}} \underline{\underline{\nabla \mathbf{u}}}+\left(\underline{\underline{\sigma}}^{\mathrm{p}} \underline{\underline{\nabla \mathbf{u}}}\right)^{T}+\alpha_{2} \phi_{a}^{2} \underline{\underline{I}}-\beta\left(\operatorname{Tr}\left(\underline{\underline{\sigma}}^{\mathrm{p}}\right) / z^{\mathrm{p}}\right) \underline{\underline{\sigma}}^{\mathrm{p}}
$$

Since the variable $z^{\mathrm{p}}$ is important in determining the local link breaking rate, it is now an actual component of the model (not just a diagnostic variable), and we follow its evolution using the equation:

$$
z_{t}^{\mathrm{p}}+\mathbf{u} \cdot \nabla z^{\mathrm{p}}=\alpha_{0} \phi_{a}^{2}-\beta\left(\operatorname{Tr}\left(\underline{\underline{\sigma}}^{\mathrm{p}}\right) / z^{\mathrm{p}}\right) z^{\mathrm{p}} .
$$

We refer to the version of the model consisting of Eqs.(18-21,28,29) as the 'approximate closure' model. 
Asymptotic and numerical analyses of the approximate closure and full multiscale models under simple shear flow show, given a breaking rate function in the multiscale model, how to choose the function $\beta\left(\operatorname{Tr}\left(\underline{\underline{\sigma}}^{\mathrm{p}}\right) / z^{\mathrm{p}}\right)$ so that the two models' behavior matches very closely for all shear rates [37]. In Fig. 7 we show a different comparison between the two models. The experiment shown is an extension of that depicted in Fig. 6. Events begin as in the earlier experiment, but after a specified time has elapsed, further activation is shut off, and extra forces are applied (at the locations indicated by the bars in Fig. 7) to accelerate the fluid and increase the stress on the aggregate in an attempt to break it into two pieces. We see that the approximate closure model did a good job of capturing the behavior of the full multiscale model in this complex situation, and we note that the closure model calculations took about $1 \%$ of the computational time of the multiscale calculation. In corresponding calculations with a constant link breaking rate, it proved impossible to break the aggregate; the central portion of the aggregate did neck off to an extent but did not break. Calculations with the full multiscale model and constant breaking rate showed that the explanation was the existence of a relatively few nonphysically long links that generated most of the force resisting rupture.
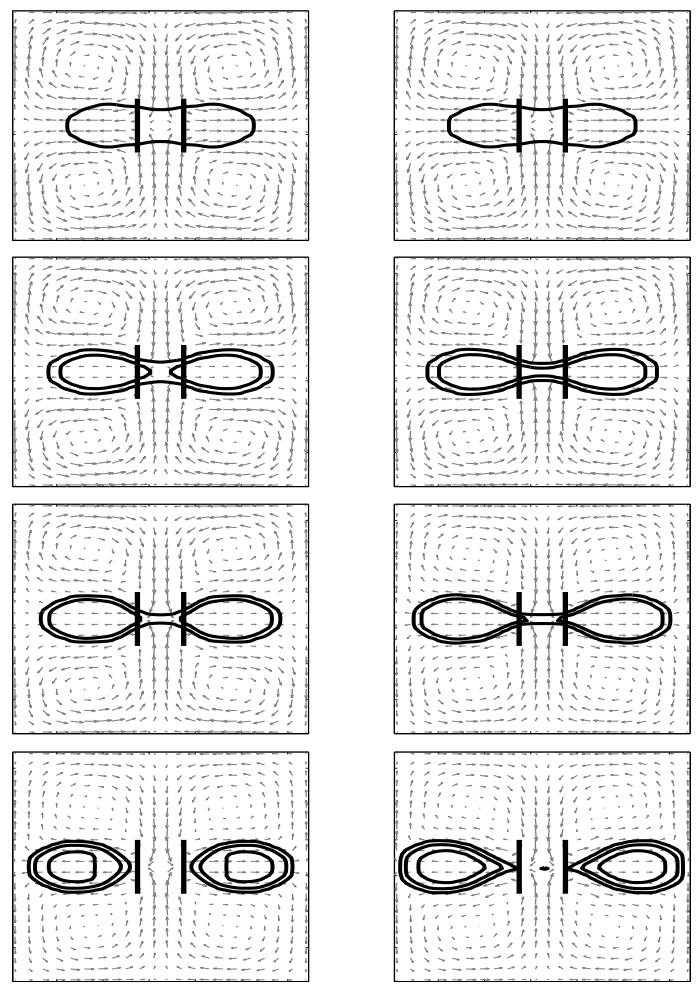

Fig. 7. Succession of snapshots of the velocity field and aggregation intensity contours for calculations with (left) the full multiscale model Eqs.(18-22) and (right) the approximate closure model Eqs.(18-21,28,29). Bars in each picture denote locations of applied pulling force. Full multiscale model computations were done as described in [38]. 


\subsection{Vessel walls in the continuum model}

To model intravascular events, the vessel wall must be modeled and the nature of platelet interactions with it must be defined. We use an IB approach to build the actual structure of the wall; that is, we use strings of IB points connected elastically to one another and to prescribed tether point locations (see section 4.1) to construct an approximately stationary vessel wall with the geometry of the vessel we wish to simulate. To define the injured portion of the wall, we introduce a density function $w(\mathbf{x}, t)$ of reactive wall sites. The function $w$ is defined everywhere in the domain, but it is nonzero only in a thin layer of space along the portion of the wall deemed injured. Platelets interact with these sites in two ways; they can be activated and they can form adhesive links where $w>0$. It is straightforward to incorporate these interactions into the model. To add the possibility of platelet activation by contact with the reactive wall sites, an additional term is added to each of the transport equations Eqs.(19)-(21) as illustrated here for the equation for $\phi_{n}$ :

$$
\left(\phi_{n}\right)_{t}+\mathbf{u} \cdot \nabla \phi_{n}=D_{n} \Delta \phi_{n}-R(c) \phi_{n}-\tilde{R}(w) \phi_{n}
$$

The new term $\tilde{R}(w) \phi_{n}$ represents activation of platelets at a per platelet rate $\tilde{R}(w)$ that is positive only where $w>0$. To add adhesive platelet-wall links to the full multiscale model, we would introduce an adhesive link function $E^{w}$ analogous to the cohesive link function $E$. Here, we skip to the analogue of the the closure approximation for $\underline{\underline{\sigma}}^{\mathrm{p}}$ and $z^{\mathrm{p}}$ and introduce the following equations for an adhesive link stress tensor $\underline{\underline{\sigma}}^{\mathrm{w}}$ and adhesive link intensity $z^{\mathrm{w}}$ :

$$
\begin{gathered}
\underline{\underline{\sigma}}_{t}^{\mathrm{w}}+\mathbf{u} \cdot \nabla \underline{\underline{\sigma}}^{\mathrm{w}}=\underline{\underline{\sigma}}^{\mathrm{w}} \underline{\underline{\nabla \mathbf{u}}}+\left(\underline{\underline{\sigma}}^{\mathrm{w}} \underline{\underline{\nabla \mathbf{u}}}\right)^{T}+\alpha_{2}^{w} \phi_{a} w \underline{\underline{I}}-\beta^{w}\left(\operatorname{Tr}\left(\underline{\underline{\sigma}}^{\mathrm{w}}\right) / z^{\mathrm{w}}\right) \underline{\underline{\sigma}}^{\mathrm{w}} \\
z_{t}^{\mathrm{w}}+\mathbf{u} \cdot \nabla z^{\mathrm{w}}=\alpha_{0}^{w} w \phi_{a}-\beta^{w}\left(\operatorname{Tr}\left(\underline{\underline{\sigma}}^{\mathrm{w}}\right) / z^{\mathrm{w}}\right) z^{\mathrm{w}} .
\end{gathered}
$$

The divergence of this stress tensor is added to the forces driving the fluid motion on the right hand side of the Navier-Stokes equations (18). The adhesive link breaking rate $\beta^{w}$ is assumed to be a function of the macroscale quantity $\operatorname{Tr}\left(\underline{\underline{\sigma}}^{\mathrm{w}}\right) / z^{\mathrm{w}}$. Eq. (31) is very similar to the equation for $\underline{\underline{\sigma}}^{\mathrm{p}}$; a difference is that the rate of formation of adhesive links depends on the product $w \phi_{a}$ since these links join reactive wall sites and platelets. The constants $\alpha_{2}^{w}$ and $\alpha_{0}^{w}$ are analogues of the constants $\alpha_{2}$ and $\alpha_{0}$ in Eqs.(28-29).

\subsection{Numerical solution of the continuum model equations}

In this section we describe the computational methods used to solve the continuum model's equations. The computational domain is a rectangular region $R$ in which we construct vessel walls using the Immersed Boundary method as 
already described. In the region between the vessel walls, we apply a spatially constant background force density $\mathbf{f}^{\mathrm{g}}$ in the $x$-direction, which, for flat walls and in the absence of platelet aggregation, would result in a parabolic velocity profile between the vessel walls.

The model's Eulerian variables are approximated in the cells of a uniform Cartesian mesh placed over $R$. We take the mesh spacing in both coordinate directions to equal $h$. Mesh points are denoted $\left(x_{j}, y_{l}\right)=((j-1 / 2) h,(l-1 / 2) h)$. Time is discretized into timesteps of size $k$. We think of the discrete velocity as being defined at time levels $t_{n+1 / 2}=(n+1 / 2) k$ and all other variables as being defined at time levels $t_{n}=n k$. We do this because the 'time-centered' velocities $\mathbf{u}^{n+1 / 2}$ are involved in the transport of advected quantities between times $t_{n}$ and $t_{n+1}$, and the 'time-centered' stresses, such as $\left(\underline{\underline{\sigma}}^{\mathrm{p}}\right)^{n}$, determine the fluid motion between times $t_{n-1 / 2}$ and $t_{n+1 / 2}$. The notation $\mathbf{u}_{j l}^{n+1 / 2}$ is used for our approximation to the velocity in the cell centered at $\left(x_{j}, y_{l}\right)$ at time $t_{n+1 / 2}$, and similar notation is used for each of the other Eulerian variables. For each of the partial differential equations which govern the behavior of an Eulerian variable, we use an appropriate finite-difference approximation defined at points of this mesh. During each timestep of the computation, we use a sequence of fractional steps to update each of the unknowns, as follows:

(1) The adhesion and cohesion force densities are calculated using discrete versions of $\mathbf{f}^{p}=\nabla \cdot \underline{\underline{\sigma}}^{\mathrm{p}}$ and $\mathbf{f}^{w}=\nabla \cdot \underline{\underline{\sigma}}^{\mathrm{w}}$ and summed to give their contributions to the fluid force density $\mathbf{f}^{n}$. The background force density $\mathbf{f}^{g}$ is also added to $\mathbf{f}^{n}$.

(2) The IB points are moved (using $\mathbf{u}^{n-1 / 2}$ ) and the IB forces are calculated and transmitted to the fluid grid adding to the fluid force density $\mathbf{f}^{n}$.

(3) Using $\mathbf{f}^{n}$, the discretized Navier-Stokes equations are solved to give new velocities $\mathbf{u}^{n+1 / 2}$ and pressure $p^{n}$.

(4) The variables $\phi_{\mathrm{a}}, \phi_{\mathrm{n}}, c, \underline{\sigma}^{\mathrm{p}}, \underline{\underline{\sigma}}^{\mathrm{w}}, z^{\mathrm{p}}$, and $z^{\mathrm{w}}$ are updated to account for advective transport (using $\mathbf{u}^{\bar{n}+1 / 2}$ ), and $\phi_{\mathrm{n}}$ and $c$ are further updated to account for diffusive transport.

(5) The variables $\phi_{\mathrm{a}}, \phi_{\mathrm{n}}, c, \underline{\underline{\sigma}}^{\mathrm{p}}, \underline{\underline{\sigma}}^{\mathrm{w}}, z^{\mathrm{p}}$, and $z^{\mathrm{w}}$ are updated to account for the reaction terms in their respective transport equations and to yield values at time level $t_{n+1}$.

Additional description of parts of the numerical methods follows; more details can be found in [39].

\subsubsection{Solution of Navier-Stokes Equations}

To solve the Navier-Stokes equations, we use a second-order approximate projection method [40]. In each timestep, the method has two substeps. In the first, a discretization of the momentum equations is used to determine an 
intermediate velocity field $\mathbf{u}^{*}$ which is typically not divergence free

$$
\frac{\mathbf{u}^{*}-\mathbf{u}^{n-1 / 2}}{k}+\mathbf{a}^{n}=-G p^{n-1}+\frac{\nu}{2}\left(L \mathbf{u}^{*}+L \mathbf{u}^{n-1 / 2}\right)+\mathbf{f}^{n} .
$$

In this equation and below, $G, D$, and $L$ are discrete gradient, divergence, and Laplacian operators defined using standard central difference approximations except close to domain boundaries. The term

$$
\mathbf{a}^{n}=\frac{3}{2}\left(\mathbf{u}^{n-1 / 2} \cdot G\right) \mathbf{u}^{n-1 / 2}-\frac{1}{2}\left(\mathbf{u}^{n-3 / 2} \cdot G\right) \mathbf{u}^{n-3 / 2}
$$

is an approximation at time $t_{n}$ to the nonlinear advection term $(\mathbf{u} \cdot \nabla) \mathbf{u}$ in the momentum equation.

In the second substep of an exact projection method, $\mathbf{u}^{*}$ would be decomposed into the sum of a divergence free velocity field $\mathbf{u}^{n+1 / 2}$ and a gradient field $G \phi$ which would be used to update the pressure

$$
\begin{aligned}
& \mathbf{u}^{*}=\mathbf{u}^{n+1 / 2}+k G \phi \\
& p^{n}=p^{n-1}+k G \phi-\frac{\nu}{2} D \cdot \mathbf{u}^{*} .
\end{aligned}
$$

The requirement $D \cdot \mathbf{u}^{n+1 / 2}=0$ used with Eq.(34) would give a discrete Poisson equation $k D \cdot G \phi=D \cdot \mathbf{u}^{*}$ with a wide (4h-by-4h) stencil. Instead, because the resulting Navier-Stokes solver has better stability properties, we solve $k L \phi=D \cdot \mathbf{u}^{*}$ with the standard 5-point discrete Laplacian. Therefore, $D$. $\mathbf{u}^{n+1 / 2}=0$ is satisfied only approximately to $O\left(h^{2}\right)$. As part of the projection step, we calculate cell-edge velocities $u_{j \pm 1 / 2, l}$ and $v_{j, l \pm 1 / 2}$ which satisfy the incompressibility equation

$$
u_{j+1 / 2, l}^{n+1 / 2}-u_{j-1 / 2, l}^{n+1 / 2}+v_{j, l+1 / 2}^{n+1 / 2}-v_{j, l-1 / 2}^{n+1 / 2}=0 .
$$

This property is important in the algorithm used to advect the Eulerian variables other than $\mathbf{u}$.

\subsubsection{Advection and diffusion}

We use a slight modification of LeVeque's high resolution advection algorithm [41] to discretize the advective terms in the transport equations for $\phi_{\mathrm{n}}, \phi_{\mathrm{a}}, c$, $\underline{\sigma}^{\mathrm{p}}, \underline{\underline{\sigma}}^{\mathrm{w}}, z^{\mathrm{p}}$, and $z^{\mathrm{w}}$. The method is second-order accurate when the solution and velocity field are smooth, and uses flux-limiters to control oscillations in the numerical solution near discontinuities or steep gradients. LeVeque's algorithm is concerned with solution of a scalar advection equation of the form 


$$
q_{t}+\mathbf{u} \cdot \nabla q=0
$$

where the velocity field $\mathbf{u}$ is incompressible. Because $\nabla \cdot \mathbf{u}=0$, the advective form Eq.(37) can also be written in conservative form as $q_{t}+\nabla \cdot(\mathbf{u} q)=0$. The two forms are equivalent for the differential equations, but discretizations based on the advective form are generally different from and often superior to those based on the conservative form. Among the advantages of advective differencing is better treatment of patches of constant $q$. On the other hand, advective differences may not preserve total mass.

In LeVeque's algorithm, $q_{j l}^{n}$ is interpreted as the cell-average of $q$ over the cell $j l$, and the method uses cell-edge velocities in defining numerical flux functions $F_{j \pm 1 / 2, l}$ and $G_{j, l \pm 1 / 2}$ which give fluxes of $q$ across the respective cell edges. The final update formula for $q$ is

$$
q_{j l}^{n+1}=q_{j l}^{n}-\frac{k}{h}\left\{F_{j+1 / 2, l}-F_{j-1 / 2, l}+G_{j, l+1 / 2}-G_{j, l-1 / 2}\right\} .
$$

The version of LeVeque's algorithm that we use proceeds in four steps, the first corresponding to a first-order upwind method, and the later steps giving a series of improvements to this basic method. Each of the steps can be described in terms of waves propagating across the edges of the cells with each wave contributing to the numerical flux of $q$ from one cell to another. The algorithm is a hybrid in that the first step is written in advective form while the correction terms, though based on advective differences, are written in terms of flux differences. The resulting algorithm has the good features of advective differencing, but is fully conservative provided the discrete incompressibility condition Eq.(36) holds.

We use LeVeque's advection algorithm over the entire grid and and we want to ensure that there is no advective flux across the immersed boundaries that make up the vessel walls. We accomplish this by employing a mask which zeros the cell edge velocities for any cell edges outside of the immersed boundary walls.

The variables $\phi_{\mathrm{n}}$ and $c$ are also affected by diffusion. We use a Crank-Nicolson time-discretization of the diffusive terms in the equations for $\phi_{\mathrm{n}}$ and $c$, with standard five-point approximations to the Laplacian operator at most grid points. For points close to the vessel walls, we use a modified stencil that enforces a no diffusive flux boundary condition at the vessel walls. The stencils are derived using the ideas of the immersed interface methods introduced by LeVeque and Li for elliptic problems with discontinuous coefficients [20], and extended to elliptic and parabolic problems with Neumann conditions imposed along an irregular boundary [19]. The combined advection-diffusion algorithm for $\phi_{\mathrm{n}}$ and $c$ is second order accurate in test problems for which the solution is smooth. 


\subsubsection{Reaction Terms}

We turn next to the reaction terms in a) Eqs.(19)-(21); b) Eqs.(28) and (29); and c) Eqs.(31) and (32). In these terms, there is no coupling between different grid points, so our discussion here applies to each grid point $\left(x_{j}, y_{l}\right)$ separately. We treat separately the reaction terms within each of the three groups notated above and describe each in turn. Platelets display a threshold-like response to activating chemicals [42], and so we take the activation rate function $R(c)$ in Eqs.(19-21) to be $R(c)=R_{0} H\left(c-c_{T}\right)$ where $H(\cdot)$ is a smoothed version of the Heaviside step function and $c_{T}$ is the threshold concentration for activation. A similar choice is made for $R^{\mathrm{w}}(w)$.

The reaction terms for $\phi_{\mathrm{n}}, \phi_{\mathrm{a}}$, and $c$ give rise to the ordinary differential equations

$$
\begin{aligned}
& \frac{d \phi_{\mathrm{n}}}{d t}=-\left(R(c)+R^{\mathrm{w}}(w)\right) \phi_{\mathrm{n}} \\
& \frac{d \phi_{\mathrm{a}}}{d t}=\left(R(c)+R^{\mathrm{w}}(w)\right) \phi_{\mathrm{n}} \\
& \frac{d c}{d t}=A\left(R\left(c^{n}\right)+R^{\mathrm{w}}(w)\right) \phi_{\mathrm{n}}(t)-K c .
\end{aligned}
$$

To update, $\phi_{\mathrm{n}}$ and $\phi_{\mathrm{a}}$, we assume that $c$, and therefore $R(c)$, is constant over the duration of the timestep and we solve analytically the resulting linear differential equations Eqs.(39) and (40) to obtain $\phi_{\mathrm{n}}^{n+1}$ and $\phi_{\mathrm{a}}^{n+1}$. Then, we replace $\phi_{\mathrm{n}}(t)$ and $\phi_{\mathrm{a}}(t)$ in Eq.(41) by their respective averages $\left(\phi_{\mathrm{n}}^{n+1}+\phi_{\mathrm{n}}^{n}\right) / 2$ and $\left(\phi_{\mathrm{a}}^{n+1}+\phi_{\mathrm{a}}^{n}\right) / 2$, and solve the resulting linear equation analytically to determine $c^{n+1}$.

The reaction terms in Eqs.(28) and (29) give rise to the ordinary differential equations

$$
\begin{aligned}
& \frac{d{\underline{\underline{\sigma^{\mathrm{p}}}}}^{\mathrm{p}}}{d t}=\underline{\underline{\sigma}}^{\mathrm{p}} \underline{\underline{\nabla \mathbf{u}}}+\left(\underline{\underline{\sigma}}^{\mathrm{p}} \underline{\underline{\nabla \mathbf{u}}}\right)^{T}+a_{2} \phi_{\mathrm{a}}{ }^{2} \underline{\underline{I}}-\beta\left(\frac{\operatorname{Tr}\left(\underline{\underline{\underline{\sigma}}}^{\mathrm{p}}\right)}{z^{\mathrm{p}}}\right) \underline{\underline{\sigma}}^{\mathrm{p}} \\
& \frac{d z^{\mathrm{p}}}{d t}=a_{0} \phi_{\mathrm{a}}^{2}-\beta\left(\frac{\operatorname{Tr}\left(\underline{\underline{\underline{\sigma}}}^{\mathrm{p}}\right)}{z^{\mathrm{p}}}\right) z^{\mathrm{p}} .
\end{aligned}
$$

These are used to determine the cohesion force density $\mathbf{f}^{\mathrm{p}}$ which contributes substantially to determining the fluid motion, and we found it important for stability to use an implicit (trapezoidal) time discretization of these equations. To describe it, let $\mathcal{A}$ be the operator which computes the time-average of its input at times $t_{n}$ and $t_{n+1}$, and let $\underline{\underline{\nabla \mathbf{u}}}=\underline{\underline{\nabla \mathbf{u}}}^{n+1 / 2}$. Our discretization is 


$$
\begin{aligned}
\frac{\left(\underline{\underline{\sigma}}^{\mathrm{p}}\right)^{n+1}-\left(\underline{\underline{\sigma}}^{\mathrm{p}}\right)^{n}}{k} & =\mathcal{A}\left(\underline{\underline{\sigma}}^{\mathrm{p}}\right) \underline{\underline{\nabla \mathbf{u}}}+\left(\mathcal{A}\left(\underline{\underline{\sigma}}^{\mathrm{p}}\right) \underline{\underline{\nabla \mathbf{u}}}\right)^{T}+a_{2} \mathcal{A}\left(\phi_{\mathrm{a}}\right)^{2} \\
& -\mathcal{A}\left(\beta\left(\frac{\operatorname{Tr}\left(\underline{\underline{\underline{\sigma}}}^{\mathrm{p}}\right)}{z^{\mathrm{p}}}\right) \underline{\underline{\sigma}}^{\mathrm{p}}\right) \\
\frac{\left(z^{\mathrm{p}}\right)^{n+1}-\left(z^{\mathrm{p}}\right)^{n}}{k} & =a_{0} \mathcal{A}\left(\phi_{\mathrm{a}}\right)^{2}-\mathcal{A}\left(\beta\left(\frac{\operatorname{Tr}\left(\underline{\underline{\sigma}}^{\mathrm{p}}\right)}{z^{\mathrm{p}}}\right) z^{\mathrm{p}}\right) .
\end{aligned}
$$

These implicit equations are solved iteratively with Newton's method, using local time-refinement at a grid point, if necessary, to ensure convergence of the iterations. The same procedure is used for the reaction terms in the equations for $\underline{\sigma}^{\mathrm{w}}$ and $z^{\mathrm{w}}$.

\subsection{Continuum model simulation results}

An important application of the continuum model is to investigate thrombosis in a stenotic (constricted) vessel following the rupture of an atherosclerotic plaque and the consequent exposure of strongly thrombogenic stimuli. Atherosclerotic plaques can develop slowly over many years and, in themselves, may cause little problem. However they are mechanically fragile and can rupture and trigger thrombosis that, in a matter of minutes, can lead to occlusion of the vessel in which the plaque resides, or can lead to the dispersal of clot fragments into the blood that end up blocking smaller vessels downstream.

Simulations in a 50\% stenosis (see [39]) show that, under some conditions, the thrombus grows to occlude the vessel. In Fig. 8, we show two simulations, identical except for the location of the rupture within the stenosis. With a rupture at the downstream end, where the flow through the stenosis is decelerating and the shear stresses are relatively low, a solid thrombus grows slowly out into the sheltered recirculation zone downstream of the plaque. With a rupture of identical size, but at the upstream end of the stenosis, where the flow is accelerating and the shear stresses are relatively high, the evolution of the thrombus is very different. As it begins to grow, the initial thrombus is subject to high stresses which lead to large strains and a high rate of cohesive link breaking (see Fig. 9). The thrombus never becomes fully solid; it behaves more as a weakly-elastic very viscous fluid. Pieces of it fragment and are carried downstream. The thrombus remains small, and eventually is broken apart (not shown) by continued shear stress. Meanwhile, the fast flow carries activating chemical through the stenosis and into the recirculating zone where a larger thrombus develops. The center of this thrombus becomes quite solid, but the thrombus is not anchored to the injured portion of the vessel wall and cannot adhere to the healthy portions of the vessel wall, so it too is eventually washed downstream. The two simulations, identical in set up except for the location of the injury relative to the flow, give a striking illustration of the 
importance of fluid dynamics in the clotting process. For these simulations, the peak inflow velocity was $10 \mathrm{~cm} / \mathrm{sec}$, the channel height and length were $0.18 \mathrm{~cm}$ and $0.8 \mathrm{~cm}$, respectively, the Reynolds number about 50, and the maximum shear rate, which occured at the upstream top corner of the stenosis, was $1750 \mathrm{sec}^{-1}$. The duration of the simulations was about $0.5 \mathrm{sec}$, which like the microscale simulations, is about two orders of magnitude too fast. 


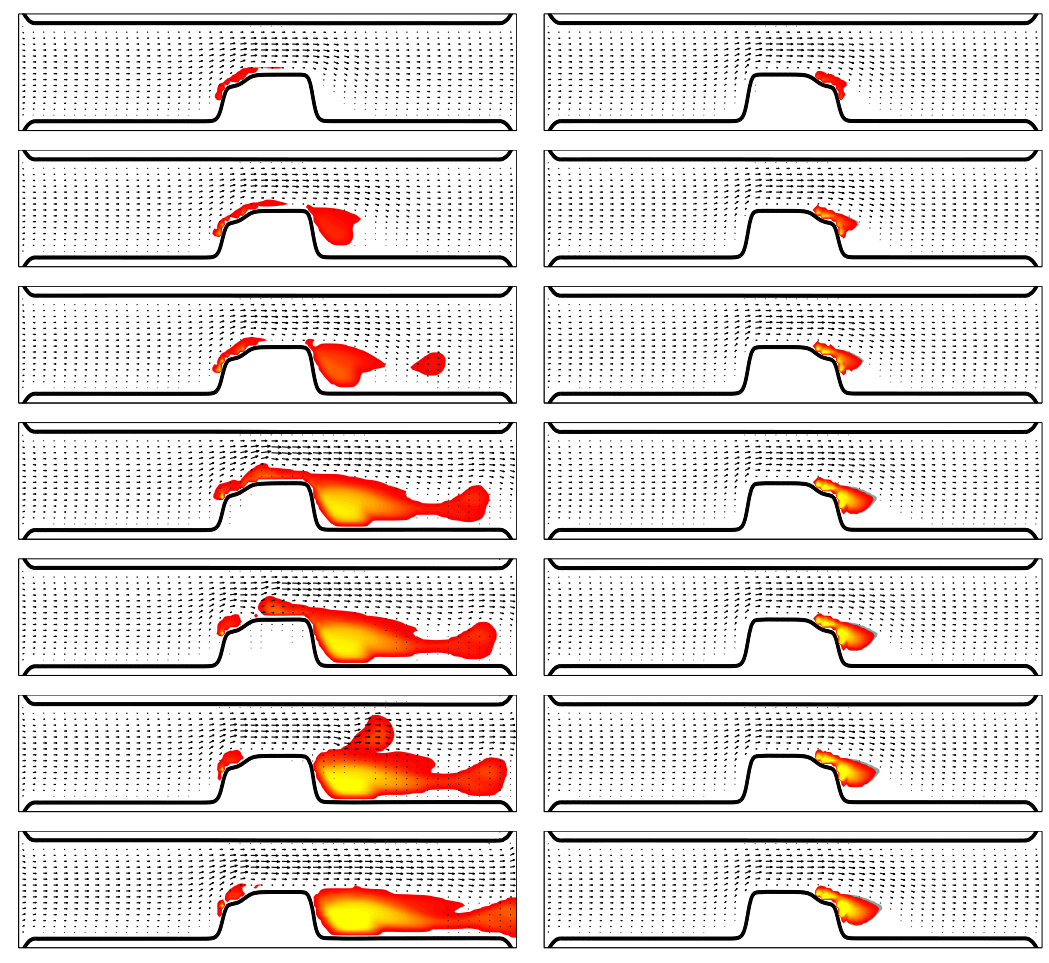

Fig. 8. Snapshots at corresponding times from two simulations of thrombosis following rupture of $50 \%$ stenosis. Flow conditions and kinetic parameters are the same for the two simulations; only the location of the rupture differs. Left: Rupture at upstream end of stenosis where flow is accelerating and shear stress is high. Right: Rupture at downstream end of stenosis where flow is slowing and shear stress is relatively low. Each plot shows velocity field and the aggregation intensity $z^{\mathrm{p}}+z^{\mathrm{w}}$ (red: low, yellow: high).
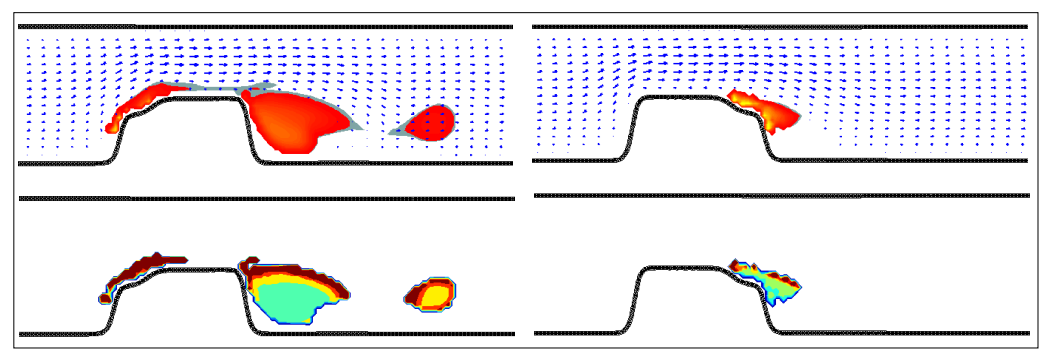

Fig. 9. Close-up snapshots at corresponding times from the simulations shown in Fig. 8. Top panel shows velocity field and aggregation intensity $z^{\mathrm{p}}+z^{\mathrm{w}}$ (red: low, yellow: high). Bottom panel shows strain in thrombi (dark: high, pale: low). 


\section{Conclusions}

We have presented both semidiscrete microscale and continuum macroscale models of platelet aggregation during blood clotting. The microscale models represent each platelet as a separate entity using Peskin's Immersed Boundary method. The continuum models are motivated by the IB idea of representing biological tissue as a composite incompressible fluid-elastic material, so that changes in geometry or phase are manifest solely through changes in mechanical forces within the material. Both types of models are able to capture salient behaviors in the platelet aggregation process including the influence of aggregate growth on the flow, shear-stress-related limitations of aggregate growth, and the possibility of complete vessel occlusion.

The implementation of the IB method is relatively straightforward, since it uses a fixed Eulerian grid, compared to other computational methods for treating deforming objects interacting with a fluid. One significant limitation in computing with the IB method is in having to use very small timesteps to maintain stability when doing explicit time-stepping. Recently, new insights have been developed into what is needed in an implicit discretization in order to achieve unconditional stability in IB calculations $[43,44]$. This may lead to the development of efficient implicit IB methods that will lessen the current timestep restrictions. For work in this direction, see [45].

There are substantial challenges remaining for both the micro- and macroscale modeling projects. Three-dimensional microscale simulations are very computationally expensive, and substantial work to make good use of new implicit solvers, parallelization, and other ideas for exploiting differences in time scales will be needed before we can simulate physiologically interesting periods of time. Simulation of the continuum model equations requires being able to handle adjacent materials of very different mechanical properties. The computational methods described in this paper do a good job in most situations, but can produce non-physical (bounded) oscillations under some circumstances. Work on algorithms that better exploit the mathematical structure of the continuum equations to eliminate this problem is described in [46]. The current model is also limited in that all species move in the same velocity field. Because there can be no relative motion between aggregated platelets and the local fluid, in order to have a solid aggregate the local fluid must be brought to rest. This means that for a solid aggregate to develop in the current model, it must do so quickly. It is more realistic to allow relative motion between the fluid and platelets in porous aggregates of low platelet volume fraction. As the volume fraction of platelets in the aggregate increases, in part because the flow brings new platelets into the thrombus, the thrombus becomes less permeable and the relative motion of fluid and platelets decreases toward zero. We are developing versions of the model that allow such relative motion between 
aggregated platelets and the local fluid.

Acknowledgments Portions of this work were done in collaboration with Drs. Nien-Tzu Wang and Haoyu Yu. 


\section{References}

[1] C. S. Peskin, The immersed boundary method, Acta Numerica 11 (2002) 479517.

[2] V. T. Turitto, H. L. Goldsmith, Rheology, transport and thrombosis in the circulation, in: J. Loscalzo, M. Creager, V. Dzau (Eds.), Textbook of Vascular Medicine, 2nd Edition, Little, Brown \& Co., New York, 1996, pp. 141-184.

[3] S. Jackson, W. Nesbitt, S. Kulkarni, Signaling events underlying thrombus formation, Journal of Thrombosis and Haemostasis 1 (2003) 1602-1612.

[4] B. Savage, E. Saldivar, Z. M. Ruggeri, Initiation of platelet adhesion by arrest onto fibrinogen or translocation on von Willebrand factor, Cell 84 (1996) 289297.

[5] R. W. Colman, P. N. Walsh, Mechanisms of platelet aggregation, in: R. W. Colman, J. Hirsh, V. J. Marder, E. W. Salzman (Eds.), Hemostasis and Thrombosis: Basic Principles and Clinical Practice, J.B. Lippincott Company, Philadelphia, 1987, pp. 594-605.

[6] J. Jesty, Y. Nemerson, The pathways of blood coagulation, in: E. Beutler, M. Lichtman, B. Coller (Eds.), Williams Hematology, 5th Edition, McGrawHill, New York, 1995, pp. 1227-1238.

[7] J. W. Weisel, Fibrinogen and fibrin, Adv Protein Chem 70 (2005) 247-299.

[8] A. L. Fogelson, N. Tania, Coagulation under flow: The influence of flow-mediated transport on the initiation and inhibition of coagulation, Pathophysiology of Haemostasis and Thrombosis 34 (2005) 91-108.

[9] R. D. Guy, A. L. Fogelson, J. P. Keener, Modeling fibrin gel formation in a shear flow, Mathematical Medicine and BiologyTo appear.

[10] A. L. Kuharsky, A. L. Fogelson, Surface-mediated control of blood coagulation: The role of binding site densities and platelet deposition, Biophysical Journal 80 (2001) 1050-1074.

[11] V. T. Turitto, H. J. Weiss, Rheological factors influencing platelet interactions with vessel surfaces, J. Rheology 23 (1979) 735.

[12] E. C. Eckstein, F. Belgacem, Model of platelet transport in flowing blood with drift and diffusion terms, Biophy J 60 (1991) 53-69.

[13] L. J. Fauci, A. L. Fogelson, Truncated Newton methods and the modelling of complex immersed elastic structures, Comm. Pure Appl. Math. 46 (1993) $787-818$.

[14] A. Fogelson, A. Kuharsky, H. Yu, Computational modeling of blood clotting: Coagulation and three-dimensional platelet aggregation, in: W. Alt, M. Chaplain, M. Griebel, J. Lenz (Eds.), Polymer and Cell Dynamics: Multicsale Modeling and Numerical Simulations, Birkhaeuser-Verlag, Basel, 2003, pp. 145-154. 
[15] A. L. Fogelson, A mathematical model and numerical method for studying platelet adhesion and aggregation during blood clotting, J. Comput. Phys. 56 (1984) 111-134.

[16] H. Yu, Three dimensional computational modeling and simulation of platelet aggregation on parallel computers, Ph.D. thesis, University of Utah (2000).

[17] R. D. Guy, A. L. Fogelson, Stability of approximate projection methods on cell-centered grids, Journal of Computational Physics 203 (2005) 517-538.

[18] P. Bagchi, Mesoscale simulation of blood flow in small vessels, Biophysical Journal 92 (2007) 1858-1877.

[19] A. L. Fogelson, J. P. Keener, Immersed interface methods for Neumann and related problems in two and three dimensions, SIAM J. Sci. Comput. 22 (2000) $1630-1654$.

[20] R. J. LeVeque, Z. Li, The immersed interface method for elliptic equations with discontinuous coefficients and singular sources, SIAM J. Numer. Anal. 31 (1994) 1019-1044.

[21] Z. Li, K. Ito, The Immersed Interface Method: Numerical Solutions of PDEs Involving Interfaces and Irregular Domains, SIAM, Philadelphia, PA, 2006.

[22] P. Lee, C. S. Peskin, personal communication.

[23] T. Doggett, G. Girdhar, A. Lawshe, D. W. Schmidtke, I. Laurenzi, S. Diamond, T. Diacovo, Selectin-like kinetics and biomechanics promote rapid platelet adhesion in flow: The GPIb $\alpha$-vWF tether bond, Biophysical Journal 83 (2002) 184-205.

[24] L. V. Parise, S. S. Smyth, B. S. Coller, Platelet morphology, biochemistry, and function, in: E. Beutler, B. Coller, M. Lichtman, T. Kipps, U. Seligsohn (Eds.), Williams Hematology, McGraw Hill, New York, 2001, pp. 1357-1408.

[25] S. Kulkarni, S. M. Dopheide, C. L. Yap, C. Ravanat, M. Freund, P. Mangin, K. A. Heel, A. Street, I. S. Harper, F. Lanza, S. P. Jackson, A revised model of platelet aggregation, Journal of Clinical Investigation 105 (2000) 783-791.

[26] M. Mazzucato, M. R. Cozzi, P. Pradella, Z. M. Ruggeri, L. De Marco, Distinct roles of ADP receptors in von Willibrand factor-mediated platelet signaling and activation under high flow, Blood 104 (2004) 3221-3227.

[27] M. Kahn, M. Hakanishi-Matsui, M. Shapiro, H. Ishihara, S. Coughlin, Proteaseactivated receptors 1 and 4 mediate activation of human platelets by thrombin, Journal of Clinical Investigation 103 (1999) 879-887.

[28] G. I. Bell, Models for the specific adhesion of cells to cells, Science 200 (1978) 618-627.

[29] G. Marguerie, T. S. Edgington, E. F. Plow, Interaction of fibrinogen with its platelet receptor as part of a multistep reaction in ADP-induced platelet aggregation, J. Biol. Chem. 255 (1980) 154. 
[30] N. A. Mody, O. Lamakin, T. Doggett, T. Diacovo, M. King, Mechanics of transient platelet adhesion to von Willebrand factor under flow, Biophysical Journal 88 (2005) 1432-1443.

[31] N. A. Mody, M. King, Three-dimensional simulations of a platelet-shaped spheroid near a wall in shear flow, Physics of Fluids 17 (2005) 113302.

[32] C. Pozrikidis, Flipping of an adherent blood platelet over a substrate, Journal of Fluid Mechanics 568 (2006) 161-172.

[33] M. Maxwell, E. Westein, W. Nesbitt, S. Giuliano, S. Dopheide, S. Jackson, Identification of a 2-stage platelet aggregatgion process mediating sheardependent thrombus formation, Blood 109 (2) (2007) 566-576.

[34] M. A. van Gestel, J. W. Heemskerk, D. W. Slaaf, V. V. Heijnen, S. O. Sage, R. S. Reneman, M. G. oude Egbrink, Real-time detection of activation patterns in individual platelets during thromboembolism in vivo: Differences between thrombus growth and embolus formation, Journal of Vascular Research 39 (2002) 534-543.

[35] A. L. Fogelson, Continuum models of platelet aggregation: Formulation and mechanical properties, SIAM JAM 52 (1992) 1089-1110.

[36] A. L. Fogelson, Continuum models of platelet aggregation: Mechanical properties and chemically-induced phase transitions, Contemp. Math. 141 (1993) 279-294.

[37] R. D. Guy, Asymptotic analysis of PTT type closures for network models with variable junction concentrations, J. Non-Newtonian Fluid Mech. 123 (2004) $223-235$.

[38] N. T. Wang, A. L. Fogelson, Computational methods for continuum models of platelet aggregation, J. Comput. Phys. 151 (1999) 649-675.

[39] A. L. Fogelson, R. D. Guy, Platelet-wall interactions in continuum models of platelet aggregation: Formulation and numerical solution, Mathematical Biology and Medicine 21 (2004) 293-334.

[40] D. L. Brown, R. Cortez, M. L. Minion, Accurate projection methods for the incompressible navier-stokes equations, Journal of Computational Physics 168 (2001) 464-499.

[41] R. J. LeVeque, High-resolution conservative algorithms for advection in incompressible flow, SIAM J. Numer. Anal. 33 (1996) 627-665.

[42] H. J. Weiss, Platelet physiology and abnormalities of platelet function (Part 1), New Engl. J. Med. 293 (1975) 531-541.

[43] Y. Mori, C. S. Peskin, Implicit second order immersed boundary methods with boundary mass, submitted for publication (2006).

[44] E. Newren, A. Fogelson, R. Guy, R. Kirby, Unconditionally stable discretizations of the immersed boundary equations, Journal of Computational PhysicsTo appear. 
[45] E. P. Newren, A. L. Fogelson, R. D. Guy, R. M. Kirby, A comparison of implicit solvers for the Immersed Boundary equations, submitted for publication (2007).

[46] R. D. Guy, A. L. Fogelson, A wave propagation algorithm for viscoelastic fluids with spatially and temporally varying properties, submitted for publication (2007). 